\title{
Segal's Gamma rings and universal arithmetic
}

\author{
Alain Connes and Caterina Consani \\ In memoriam Michael Atiyah, with profound admiration
}

\begin{abstract}
Segal's $\Gamma$-rings provide a natural framework for absolute algebraic geometry. We use Almkvist's global Witt construction to explore the relation with J. Borger $\mathbb{F}_{1}$-geometry and compute the Witt functor-ring $\mathbb{W}_{0}(\mathbb{S})$ of the simplest $\Gamma$-ring $\mathbb{S}$. We prove that it is isomorphic to the Galois invariant part of the BC-system, and exhibit the close relation between $\lambda$-rings and the Arithmetic site. Then, we concentrate on the Arakelov compactification $\overline{\text { Spec } \mathbb{Z}}$ which acquires a structure sheaf of $\mathbb{S}$-algebras. After supplying a probabilistic interpretation of the classical theta invariant of a divisor $D$ on $\overline{\operatorname{Spec} \mathbb{Z}}$, we show how to associate to $D$ a $\Gamma$-space that encodes, in homotopical terms, the Riemann-Roch problem for $D$.
\end{abstract}

\section{Introduction}

Michael Atiyah was quite right stating that "without dreams there is no art, no mathematics no life". Dreaming new paths in mathematics has helped mathematicians to overcome seemingly impossible technical obstacles, frequently guiding them to discover fascinating new theories. It has brought us to explore the enchanted realm of J. Tits's "field with 1 element" [35] and to develop in [21] the theory of the "absolute point" $\operatorname{Spec}\left(\mathbb{F}_{1}\right)$ and the algebraic geometry over it (speculated in [32]). This is the topic pursued in this article that we heartily dedicate to M. F. Atiyah in honor of his farsighted intuition and ability to communicate enthusiasm while spreading new ideas.

In his seminal paper [35], Tits, motivated by his geometric interpretation of C. Chevalley's work on algebraic groups of Lie type, advocated the search of a mysterious "field with one element" $\mathbb{F}_{1}$ (denoted $K_{1}$ in that paper), that would also account for the understanding of the infinite series of simple alternating groups $\operatorname{Alt}(n)(n \geq 5)$, as $\mathrm{SL}_{n}\left(\mathbb{F}_{1}\right)$ [27]. In the last thirty years, this search was pursued in a number of ways: we refer, in particular, to [4, 24,27,34,36]. From a quite different perspective, the algebraic $K$-theory of spaces initiated by $\mathrm{F}$. Waldhausen led to the notion of "brave new rings" thus shifting the interest of algebraic topologists from rings to ring spectra, where the integers $\mathbb{Z}$ become an algebra over the sphere spectrum [26]. A suitable model for connective spectra was provided by G. Segal's $\Gamma$-spaces which, endowed with Lydakis' smash product, form a closed, symmetric and monoidal category. This category is the

2010 Mathematics Subject Classification 19D55, 13F35, 14G40; 18G55; 18G30; 19L20

Keywords: Gamma-ring, Adams operations, lambda-rings, Site, Arithmetic, Arakelov compactification, Theta dimension

We are grateful to Jens Hemelaer for his useful comments on an earlier version of this paper. 


\section{Alain Connes and Caterina Consani}

natural framework for both Hochschild and cyclic homologies [23]. In our research, we found that the theory of discrete Segal's $\Gamma$-rings provides a natural extension of the classical theory of rings and semirings, hence supplying a convincing algebraic incarnation for the $\mathbb{F}_{1}$-dream of a characteristic-free (universal) theory. To be more specific, a Segal's discrete $\Gamma$-ring (we abbreviate it as $\Gamma$-ring for simplicity) is by definition a monoidal object in the category $\mathbb{S}$ - Mod of pointed covariant functors from finite pointed sets to pointed sets. In order to work with a small category one uses the skeleton category $\Gamma^{\mathrm{op}}$ with one object $n_{+}=\{0, \ldots, n\}$ ( 0 is the base point) for each integer $n . \mathbb{S}$ - Mod is a closed, symmetric and monoidal category, thus the monoids in it give a very concrete notion of $\Gamma$-rings, forming the category $\mathfrak{S}$. The simplest $\Gamma$-ring $\mathbb{S}$ corresponds to the identity functor and it is the most basic incarnation of the sphere spectrum over which the ring of integers becomes an algebra (we shall use freely the terminology "S-algebras" in place of $\Gamma$-rings).

Many attempts to model Tits' alleged "field with one element" find naturally their place among $\Gamma$-rings [19]. This holds for N. Durov's theory [24] and for the constructions with hyperrings, while both the category $\mathfrak{M o}$ of commutative pointed monoids (advocated in $[22,28,29,36]$ ), and the category of semirings (that we used extensively in [14-18] to model Tits' idea of "characteristic one") are full subcategories of $\mathfrak{S}$. The connection with the ideas developed by Kapranov and Smirnov in [27] is provided by the fact that $\mathbb{S}-$ Mod can be seen as the category of vector spaces over $\mathbb{F}_{1}$ as in op.cit. , provided one works in the presheaf topos $\Gamma^{\wedge}$ on Segal's category $\Gamma$, rather than in the topos of sets [20].

The relation with the original constructions of C. Soulé [34] came as a pleasant surprise. Our earlier developments [7] promoted, after the initial approach in [8], a theory of schemes (of finite type) over $\mathbb{F}_{1}$ using the category $\mathfrak{M} \mathfrak{R}$ obtained by gluing together the category $\mathfrak{M o}$ with the category $\mathfrak{R i n g}$ of commutative rings. In [21] we realized that the category $\mathfrak{M} \mathfrak{R}$ is simply the full subcategory of $\mathfrak{S}$ whose objects are either in $\mathfrak{M o}$ or $\mathfrak{R i n g}$ ! Thus, the a priori artificial process of glueing $\mathfrak{M} \mathfrak{R}=\mathfrak{R i n g} \cup_{\beta, \beta^{*}} \mathfrak{M o}$ described in [7] is now fully justified and has suggested to us, in view of the results of op.cit. the development of algebraic geometry working directly in $\mathfrak{S}$.

This is the path we have started to follow in [21], as far as the affine case is concerned, by extending to the general case of an arbitrary $\mathbb{S}$-algebra $A$, the construction of its spectrum as a topos $\operatorname{Spec}(A)$, endowed with a structure sheaf of $\mathbb{S}$-algebras. The spectrum $\mathfrak{S p e c}(A)$ derives from a Grothendieck site endowed with a presheaf of $\mathbb{S}$-algebras: this datum is more refined than that of the associated topos and sheaf. When $A=H R$ for a semiring $R$, the associated topos-spectrum determines the prime spectrum, while it hands Deitmar's spectrum [22] when $A=\mathbb{S} M$ is the spherical algebra associated to a monoid $M$. It is by now clear that our first development [7] is a special case of this new "absolute algebraic geometry", and in particular that $\mathfrak{S p e c}(\mathbb{S})$ is the natural candidate for $\operatorname{Spec}\left(\mathbb{F}_{1}\right)$.

The distinctive feature of this new theory is to give significance to operations which are meaningless in ordinary algebraic geometry, such as taking the quotient of a ring by a subgroup of its multiplicative group, or the restriction to the unit ball in normed rings. These operations make sense in full generality for $\mathbb{S}$-algebras, thus one can analyze their effect on the associated spectra. The connection between this development and the theory of Töen-Vaquié [36] (a general theory of algebraic geometry for any symmetric monoidal closed category that is complete and cocomplete, like the category $\mathbb{S}$ - Mod) shows that the theory in [36], when implemented to the category $\mathbb{S}$ - Mod, does not agree with ordinary algebraic geometry, already in the simplest case of the two point space corresponding to the spectrum of the product of two fields.

In the present paper we examine the connection of [21] with J. Borger's $\mathbb{F}_{1}$-theory [4]. In Section 
2, we compute the global Witt ring, as understood by Almkvist in [1,2], of $\mathbb{S}$ and we show that it is the $\lambda$-ring obtained from the integral BC-system by taking the fixed points of the Galois action of $\hat{\mathbb{Z}}^{\times}$

THEOREM 1.1. The global Witt ring $\mathbb{W}_{0}(\mathbb{S})$ is canonically isomorphic to the invariant part $\mathbb{Z}[\mathbb{Q} / \mathbb{Z}]^{G}$ of the group ring $\mathbb{Z}[\mathbb{Q} / \mathbb{Z}]$, under the action of the group $G=\operatorname{Aut}(\mathbb{Q} / \mathbb{Z})=\widehat{\mathbb{Z}}^{\times}$.

This result is in agreement with the idea developed in [10] that the integral BC-system is the Witt ring of the algebraic closure of $\mathbb{F}_{1}$. In fact we conjecture that the above Witt construction applied to $\mathbb{S}\left[\mu_{\infty}\right]$, where $\mu_{\infty}$ denotes the group of abstract roots of unity with zero added, is canonically isomorphic to the full BC-system. The key idea advocated in [4] is that $\lambda$-rings are obtained by extension of scalars from $\mathbb{F}_{1}$ to $\mathbb{Z}$. The basic result which greatly simplifies the definition of $\lambda$-rings is due to C. Wilkerson [38] and formulates, in the flat case, the structure in terms of the Adams operations and Frobenius lifts, in place of the $\lambda$-operations themselves, whose algebraic rules are more complicated to state. There is a rather obvious relation between this formulation of $\lambda$-rings and the Arithmetic Site [14] that we discuss in $\S 2.3$. Namely, a $\lambda$-ring automatically generates, using the Adams operations, a sheaf of rings over the Arithmetic topos $\widehat{\mathbb{N}^{x}}$. In Section 2, we also recall the origin of $\widehat{\mathbb{N}^{x}}$ from the cyclic and epicyclic categories and we discuss the interrelations of the various toposes candidates for the "absolute geometric point". Global fields are either function fields in finite characteristic or number fields. The analogy between these two families breaks down at the archimedean places for the lack of an "absolute arithmetic" allowing to treat them on the same footing as the ultrametric places. The simplest case to consider is the field $\mathbb{Q}$ of the rational numbers. At a non-archimedean place $p$, the subset $\left\{x \in \mathbb{Q}_{p}|| x \mid \leq 1\right\}$ is a subring of the local field $\mathbb{Q}_{p}$ but the corresponding set, at the archimedean place, does not define a subring of the local field $\mathbb{R}$. It does however still define an $\mathbb{S}$-subalgebra of $H \mathbb{R}$. In [19] we applied the theory of $\mathbb{S}$-algebras to extend the structure sheaf of Spec $\mathbb{Z}$ to the Arakelov compactification $\overline{\mathrm{Spec} \mathbb{Z}}$, as a sheaf of $\mathbb{S}$-algebras. The remaining sections of this paper concentrate on the Arakelov compactification $\overline{\operatorname{Spec} \mathbb{Z}}$ as an arithmetic curve over $\mathbb{S}$ (in fact more precisely over $\mathbb{S}\left[\mu_{2}\right]$ ). Each Arakelov divisor provides a natural sheaf of modules over this extended structure sheaf of $\overline{\text { Spec } \mathbb{Z}}$. Moreover, this new structure for $\overline{\text { Spec } \mathbb{Z}}$ over $\mathbb{S}$ endorses also a one parameter group of weakly invertible sheaves, whose tensor product rules are the same as the composition rules of Frobenius correspondences over the Arithmetic Site [13,14].

For a function field with field of constants $\mathbb{F}_{q}$, the dimension $\operatorname{dim}_{\mathbb{F}_{q}} H^{0}(D)$ of the $\mathbb{F}_{q^{-} \text {-vector space }}$ $H^{0}(D)$ of solutions of the Riemann-Roch problem for a divisor $D$ is the $\operatorname{logarithm} \log _{q}\left(\# H^{0}(D)\right)$ of the cardinality of $H^{0}(D)$. Following the analogy between function fields and number fields, $\operatorname{dim}_{\mathbb{F}_{q}} H^{0}(D)$ is replaced, in the number field case, by the theta dimension $h_{\theta}^{0}(D)$ of an Arakelov divisor $D[5,37]$. In Section 3 we introduce, in view of the new understanding of $\overline{\text { Spec } \mathbb{Z}}$ over $\mathbb{S}$, the probabilistic interpretation of $h_{\theta}^{0}(D)$, as the logarithm of an average value of the naive counting of solutions formulated in terms of $\mathbb{S}$-modules. More precisely, for a given rank one discrete subgroup $L$ in a one dimensional complex vector space $E$, one measures the length of vectors $\xi \in E$ by comparing $\xi$ with the unit of length provided by $L$. This process yields the non negative integer

$$
[\xi / L]:=\#\{\ell \in L \mid\|\ell\| \leq\|\xi\|\}
$$

that is independent of the choice of the hermitian norm on $E$. Then, in Theorem 3.3 we show the equality

$$
\exp \left(h_{\theta}^{0}(D)\right)=\int[\xi / L] d p(\xi)
$$


where the probability measure $d p(\xi)$ is a Gaussian and depends on the choice of the hermitian norm on $E$ through the normalization condition $\int\|\xi\| d p(\xi)=\frac{1}{2}$. Our probabilistic interpretation of $h_{\theta}^{0}(D)$ is implicit in some proofs of inequalities involving the naive counting. On the other hand, it differs substantially from the related work of J. B. Bost [5] based on the thermodynamic expression of the logarithm of a sum as a supremum involving the entropy function. The role of the Gaussian on the complex vector space $E$ also suggests the existence of a possible relation with the formulas of V. Mathai and D. Quillen [33] for the Chern character.

In [20] we found that the $\Gamma$-ring arising as the stalk of the structure sheaf of $\overline{\mathrm{Spec} \mathbb{Z}}$ at the archimedean place, is intimately related to hyperbolic geometry and Gromov's norm. This development involves a generalization of the homology of a simplicial complex when the coefficients are no longer abelian groups but $\mathbb{S}$-modules. This new homology takes values in $\mathbb{S}$-modules and strictly extends the standard theory. Here, the key fact is that the category of $\Gamma$-spaces, that is the central tool of [23] and forms the core of the local structure of algebraic $K$-theory, is the category of simplicial objects in $\mathbb{S}$ - Mod. Thus, according to the general principle inherent to the Dold-Kan correspondence, homotopy theory of $\Gamma$-spaces ought to be considered as absolute homological algebra of $\mathbb{S}$-modules. Since the category $\mathbb{S}-\operatorname{Mod}$ is not abelian, the tools of homological algebra need to be replaced along the line of the Dold-Kan correspondence, that provides, for an abelian category $\mathcal{A}$ the correspondence between chain complexes in non-negative degrees and simplicial objects i.e. objects of the category $\mathcal{A}^{{ }^{\mathrm{op}}}$. While in $[23]$ the systematic use of fibrant replacements frequently simplifies constructions at the price of neglecting important information, for our needs it is necessary to work directly with $\mathbb{S}$-modules rather than abelian groups, as the most genuine receptacle of homotopy invariants. This was the strategy already adopted in [20].

Therefore the natural goal to pursue, in parallel with the elaboration of "absolute algebraic geometry", is the construction of the homological algebra over $\mathfrak{S}$. The clear advantage of working with $\Gamma$-rings is that this category forms the natural groundwork where cyclic homology is rooted [23] and in particular, where de Rham theory is naturally available.

In Section 4 we construct a first instance of the analogue of the Dold-Kan correspondence for $\mathbb{S}$ modules. We consider the adelic proof of the Riemann-Roch formula as developed by A. Weil for global fields of positive characteristic. The analogue of his construction for the field $\mathbb{Q}$ provides one with a short complex $C(D)$ of $\mathbb{S}$-modules associated to an Arakelov divisor $D$ on $\overline{\operatorname{Spec} \mathbb{Z}}$. This complex arises from a natural morphism of $\mathbb{S}$-modules canonically associated to $D$ (we refer to $\S 4.2$ for its definition). In Proposition 4.9, we construct the $\Gamma$-space $\mathbf{H}(D)$ associated to the complex $C(D)$ and hence to the Arakelov divisor $D$. We view $\mathbf{H}(D)$ as the homological incarnation of the Riemann-Roch problem for $D$ and in $\S \S 4.5$ and 4.6 we determine its homotopy. It is an interesting open question to find the analogue of Serre's duality in this context, connecting (a version of) $\pi_{0}(\mathbf{H}(D))$ with $\pi_{1}(\mathbf{H}(-D))$. Obviously, the goal is to prove the Riemann-Roch formula $h_{\theta}^{0}(D)-h_{\theta}^{0}(-D)=\operatorname{deg}(D)$ for the probabilistic theta dimension in purely geometric terms, without appealing to the analytic Poisson's formula.

\section{The global Witt ring $\mathbb{W}_{0}(\mathbb{S})$}

We recall that $\mathbb{S}$-modules (equivalently $\Gamma$-sets) are by definition covariant functors $\Gamma^{\text {op }} \longrightarrow \mathfrak{S e t s}_{*}$ between pointed categories. Here, $\Gamma^{\mathrm{op}}$ is the small, full subcategory of the category $\mathfrak{F i n}_{*}$ of finite pointed sets, whose objects are the pointed sets $k_{+}:=\{0, \ldots, k\}$, for each integer $k \geq 0(0$ is the base point) and the morphisms connecting two objects are the sets $\Gamma^{\mathrm{op}}\left(k_{+}, m_{+}\right)=\{f$ : 
$\{0,1, \ldots, k\} \rightarrow\{0,1, \ldots, m\} \mid f(0)=0\}$. The morphisms in the category $\mathbb{S}-$ Mod of $\mathbb{S}$-modules are natural transformations. The category $\mathbb{S}-$ Mod is a closed, symmetric and monoidal category. An $\mathbb{S}$-algebra is a monoid in $(\mathbb{S}-\operatorname{Mod}, \wedge, \mathbb{S})$ : the natural inclusion $\mathbb{S}: \Gamma^{\text {op }} \longrightarrow \mathfrak{S e t s}_{*}$ is the simplest example.

In this section we compute Almkvist's invariant $\mathbb{W}_{0}[1,2]$ for the simplest $\mathbb{S}$-algebra, i.e. we determine $\mathbb{W}_{0}(\mathbb{S})$, motivated by the guess in $[4]$ that $W\left(\mathbb{F}_{1}\right)=\mathbb{Z}$.

\subsection{Almkvist's invariant $\mathbb{W}_{0}(\mathbb{S})$}

In Almkvist's original definition of the invariant $\mathbb{W}_{0}(A)=\tilde{K}_{0}($ end $\mathbf{P}(A))$ for a commutative ring $A$, one uses exact sequences to derive the notion of additive invariants for endomorphisms of finitely generated projective $A$-modules in the Grothendieck group $K_{0}$ (end $\left.\mathbf{P}(A)\right)$. In particular, the condition stating that the invariants are additive on exact sequences suffices to eliminate the non-trivial part of the Jordan decomposition of a matrix, thus it plays an essential role in the construction. By definition, $\mathbb{W}_{0}(A)$ is the quotient of $K_{0}($ end $\mathbf{P}(A))$ by the subgroup $K_{0}(A)$ of trivial endomorphisms. In the context of the non abelian category of $\mathbb{S}$-modules a generalization of Almkvist's construction is still possible. It suffices to require the additivity for exact sequences of endomorphisms of $\mathbb{S}$-modules of the form

$$
* \rightarrow T(E) \rightarrow E \rightarrow E / T(E) \rightarrow * \quad \forall T \in \operatorname{End}(E),
$$

where the $\mathbb{S}$-module $E / T(E)$ is defined by collapsing the $\mathbb{S}$-submodule $T(E)$ of the $\mathbb{S}$-module $E$ according to the following general process. We let $\mathfrak{S e t s}_{2, *}$ be the category of pairs $X \supset Y$ of pointed sets (here $* \in Y \subset X$ ) where morphisms are maps of pointed sets $f: X \rightarrow X^{\prime}$ such that $f(Y) \subset Y^{\prime}$. The collapsing of $Y$ to the base point $*$ defines a functor $\mathcal{C}: \mathfrak{S e t s}_{2, *} \longrightarrow \mathfrak{S e t s}_{*}$, $\mathcal{C}(X, Y)=X / Y$.

Definition 2.1. Let $F$ be an $\mathbb{S}$-submodule of an $\mathbb{S}$-module $E$. Then the $\mathbb{S}$-module $E / F$ is the composition of the pair $(E, F)$ with the functor $\mathcal{C}: E / F\left(k_{+}\right):=E\left(k_{+}\right) / F\left(k_{+}\right), \forall k \in \mathbb{N}$.

Next, we notice that each term of the sequence (3) is globally invariant for the action of the endomorphism $T$ and the induced action on the right term of the sequence is null i.e. its range is reduced to $*$. Since one divides as in [2] by null endomorphisms, this means that the right term of (3) is null. Therefore, in this context, it is natural to define additive invariants as follows

Definition 2.2. An additive invariant on a class $\mathcal{C}$ of $\mathbb{S}$-modules stable under the operation defined in Lemma 2.1, is a map $\chi: \operatorname{End}(\mathcal{C}) \rightarrow G$ from endomorphisms of objects of $\mathcal{C}$ to an abelian group $G$ that satisfies the following conditions

(i) $\chi(E, T)=\chi(T(E), T)$

(ii) $\chi\left(\left(E_{1} \vee E_{2}, T_{1} \vee T_{2}\right)=\chi\left(E_{1}, T_{1}\right)+\chi\left(E_{2}, T_{2}\right)\right.$.

In this paper we consider the simplest of such class $\mathcal{C}$, namely the collection of $\mathbb{S}$-modules of the form $\mathbb{S}[F]:=\mathbb{S} \wedge F$ where $F$ is a finite pointed set. Any such $\mathbb{S}$-module is a finite sum (under the join operation $\vee$ ) of copies of $\mathbb{S}$.

We denote by $\mathbb{W}_{0}(\mathbb{S})$ the abelian group range of the universal additive invariant $\tau(E, T)$ for this class $\mathcal{C}$ of $\mathbb{S}$-modules. The smash product in $\operatorname{End}(\mathcal{C})$ is defined as follows

$$
\left(E_{1}, T_{1}\right) \wedge\left(E_{2}, T_{2}\right):=\left(E_{1} \wedge E_{2}, T_{1} \wedge T_{2}\right)
$$


(where the $\wedge$ on the right of the above formula denotes the external smash). One derives a commutative ring structure on $\mathbb{W}_{0}(\mathbb{S})$.

Given an endomorphism $T: F \rightarrow F$ of a finite pointed set, we denote by $\operatorname{tr}(T)$ (the trace of $T$ ) the number of fixed points of $T$ minus one (that accounts for discarding the base point as a trivial fixed point). The subsets $T^{n}(F) \subseteq F$ obtained by iterating the endomorphism $T n$-times, define a decreasing filtration of $F$ which stabilizes, after finitely many steps, to $T^{\infty}(F) \subseteq F$.

Notice that for a permutation $T: F \rightarrow F$ in $\mathcal{C}$, the map $\mathbb{N} \ni n \mapsto T^{n}$ from non-negative integers to iterates of $T$ extends by periodicity to the profinite completion of the integers $\hat{\mathbb{Z}}:=\lim _{n} \mathbb{Z} / n \mathbb{Z}$. In this case, we shall use freely the symbol $n \mapsto T^{n}$ for $n \in \hat{\mathbb{Z}}$. In the following, we identify the compact abelian group $\hat{\mathbb{Z}}$ with the dual of the discrete abelian group $\mathbb{Q} / \mathbb{Z}$. More specifically, we first use the ring isomorphism $\hat{\mathbb{Z}} \simeq \operatorname{End}(\mathbb{Q} / \mathbb{Z})$ mapping an integer $n \in \mathbb{Z} \subset \hat{\mathbb{Z}}$ to the endomorphism of multiplication by $n$ in $\mathbb{Q} / \mathbb{Z}$. Then, we associate to $n \in \hat{\mathbb{Z}} \simeq \operatorname{End}(\mathbb{Q} / \mathbb{Z})$ the character of the discrete group $\mathbb{Q} / \mathbb{Z}$ obtained by composition with the canonical character $\exp (2 \pi i \bullet): \mathbb{Q} / \mathbb{Z} \rightarrow S^{1}$. One derives the duality pairing

$$
\langle s, n\rangle:=\exp (2 \pi i n s), \quad \forall s \in \mathbb{Q} / \mathbb{Z}, n \in \hat{\mathbb{Z}} \simeq \operatorname{End}(\mathbb{Q} / \mathbb{Z}) .
$$

Next result determines the ring $\mathbb{W}_{0}(\mathbb{S})$ for the chosen class $\mathcal{C}$ of $\mathbb{S}$-modules of the form $E=$ $\mathbb{S}[F]:=\mathbb{S} \wedge F$, with $F$ a finite pointed set.

TheOREM 2.3. The ring $\mathbb{W}_{0}(\mathbb{S})$ is canonically isomorphic to the invariant part of the group ring $\mathbb{Z}[\mathbb{Q} / \mathbb{Z}]$ for the action of the group $\operatorname{Aut}(\mathbb{Q} / \mathbb{Z})=\widehat{\mathbb{Z}}^{\times}$.

The universal additive invariant $\tau: \mathcal{C} \rightarrow \mathbb{Z}[\mathbb{Q} / \mathbb{Z}]$ is uniquely determined by the equality of Fourier transform

$$
\overline{\tau(E, T})(n)=\operatorname{tr}\left(T_{\mid T^{\infty}\left(E\left(1_{+}\right)\right)}^{n}\right), \quad \forall n \in \hat{\mathbb{Z}} .
$$

Proof. The evaluation $E \mapsto E\left(1_{+}\right)$determines a functor $\mathbb{S}-\operatorname{Mod} \longrightarrow \mathfrak{S e t s}_{*}$ and when applied to objects of $\mathcal{C}$ of the form $E=\mathbb{S}[F]:=\mathbb{S} \wedge F$, the evaluation gives finite pointed sets. This functor transforms joins $E_{1} \vee E_{2}$ to joins $F_{1} \vee F_{2}$ and maps the external smash product of objects of $\mathcal{C}$ into the smash product of pointed sets (within the category of $\mathbb{S}$-modules one has $\left.\left(F_{1} \wedge \mathbb{S}\right) \wedge\left(F_{2} \wedge \mathbb{S}\right)=\left(F_{1} \wedge F_{2}\right) \wedge \mathbb{S}\right)$. In order to show that $\tau$ defines an additive invariant, whose range is inside $\mathbb{Z}[\mathbb{Q} / \mathbb{Z}]$, we can work at the level of the finite pointed sets $F=E\left(1_{+}\right)$. The right hand side of (5) defines, by construction, an additive map to functions on $\hat{\mathbb{Z}}$ that is also multiplicative. The restriction of $T$ to $T^{\infty}(F)$ is a bijection preserving the base point, and thus admits a canonical decomposition as a sum of cyclic permutations of the form $C(k):=\left((\mathbb{Z} / k \mathbb{Z})_{+}, x \mapsto x+1\right)$, where one adds a base point fixed under $T$. Let $e: \mathbb{Q} / \mathbb{Z} \rightarrow \mathbb{Z}[\mathbb{Q} / \mathbb{Z}]$ be the canonical map from the additive group $\mathbb{Q} / \mathbb{Z}$ to its group ring, then we claim that (5) holds if one sets

$$
\tau(C(k)):=\sum_{k \gamma=0} e(\gamma) \in \mathbb{Z}[\mathbb{Q} / \mathbb{Z}] .
$$

Indeed, using (4) the Fourier transform $\tau \overline{(E, T})(n)$ is given by

$$
\tau \overline{(E, T})(n)=\sum_{k \gamma=0}\langle\gamma, n\rangle=\sum_{k s=0} \exp (2 \pi i n s)= \begin{cases}k & \text { if } n \in k \hat{\mathbb{Z}} \\ 0 & \text { otherwise. }\end{cases}
$$

Thus one gets (5) since the trace of the powers of the cyclic permutation of order $k$ is $k$ if $n \in k \hat{\mathbb{Z}}$ and 0 otherwise. This argument shows that $\tau$ is an additive invariant of $\mathcal{C}$ with values in $\mathbb{Z}[\mathbb{Q} / \mathbb{Z}]$. In fact, it also proves that $\tau$ is multiplicative, because the Fourier transform is an injective ring homomorphism from the group ring $\mathbb{Z}[\mathbb{Q} / \mathbb{Z}]$ to the algebra $C(\hat{\mathbb{Z}})$ of continuous functions on the 
compact space $\hat{\mathbb{Z}}$. It remains to show that $\tau$ is injective and surjective.

Let $E=\mathbb{S} \wedge F$ be an object of $\mathcal{C}$ and $T$ an endomorphism of $E$. Lemma 2.1 of [21] shows that for $X$ an $\mathbb{S}$-module, the map associating to $x \in \operatorname{Hom}_{\mathbb{S}}(\mathbb{S}, X)$ its value $x\left(1_{+}\right)(1) \in X\left(1_{+}\right)$ at $1 \in 1_{+}$defines a bijection of sets $\operatorname{Hom}_{\mathbb{S}}(\mathbb{S}, X) \stackrel{\sim}{\rightarrow} X\left(1_{+}\right)$. Since $E$ is a finite sum of copies of $\mathbb{S}$, any endomorphism of $E$ is thus of the form $\operatorname{Id} \wedge T$ where $T: F \rightarrow F$ is a map of finite pointed sets. Hence the evaluation functor $(E, T) \mapsto\left(E\left(1_{+}\right), T_{\mid E\left(1_{+}\right)}\right)$is faithful. Moreover, the replacement $(E, T) \mapsto(T(E), T)$ corresponds to the replacement $(F, T) \mapsto(T(F), T)$ and by $(i)$ of Definition 2.2 one has the equivalence $(F, T) \simeq\left(T^{\infty}(F), T\right)$. Thus, to show that $\tau$ is injective it is enough to prove that if $\left(F_{j}, T_{j}\right), j=1,2$, are two permutations of finite pointed sets such that $\tau\left(F_{1}, T_{1}\right)=\tau\left(F_{2}, T_{2}\right)$ then they are isomorphic. In fact, it suffices to show that the number $m(k)$ of cycles of length $k$ is the same in both permutations for any integer $k$. Observe that the number $m(k)$ is given in terms of $\tau \overline{(E, T)}(n)$ and the Möbius function $\mu$ by the equality (that follows by inverting the relation $\left.\left.\sum_{u \mid n} m(u)=\tau \overline{(E, T}\right)(n)\right)$

$$
\left.m(k)=\sum_{d \mid k} \mu(k / d) \tau \overline{(E, T}\right)(d) .
$$

We have thus shown that the number $m(k)$ is determined by $\tau(E, T)$ and hence, as explained above, that $\tau$ is injective. To show the surjectivity of $\tau$, it is enough to check that the generators of the abelian group $\mathbb{Z}[\mathbb{Q} / \mathbb{Z}]^{G}, G=\operatorname{Aut}(\mathbb{Q} / \mathbb{Z})$, are in the range of $\tau$. It is well known that a system of generators of that group is given by the elements $\rho(n):=\sum_{\gamma \in P(n)} e(\gamma)$, where $P(n) \subset$ $\{\gamma \in \mathbb{Q} / \mathbb{Z} \mid n \gamma=0\}$ is the subset of primitive roots. In view of the relation

$$
\rho(n)=\sum_{d \mid k} \mu(k / d) \tau(C(d)),
$$

obtained by inverting the relation

$$
\sum_{u \mid n} \rho(u)=\tau(C(n))
$$

one derives that $\tau$ is surjective.

Formula (6) expresses the fact that $\tau$ associates to an endomorphism $T$ in $\mathcal{C}$ its "trace" $\tau(T)$ viewed as the sum of its abstract eigenvalues. The Fourier transform $\tau \overline{(E, T)}(n)$ as in (5) plays then the role of generalized ghost component: $\overline{\tau(E, T})(n)=\operatorname{gh}_{n}((E, T))$, following the classical description of the global Witt ring.

The Frobenius endomorphisms and the Verschiebung maps are meaningful operators in $\mathbb{W}_{0}(\mathbb{S})$. The Frobenius endomorphism $F_{n}$ is defined by the equality $F_{n}((E, T)):=\left(E, T^{n}\right)$. The Verschiebung map $V_{n}$ replaces the pair $(E, T)$ by the endomorphism of the sum $\vee^{n} E$ of $n$ copies of $E$ that cyclicly permutes the terms and uses $T: E \rightarrow E$ to pass from the last term to the first. In particular, when applied to a cyclic permutation of order $m$ this process yields a cyclic permutation of order $\mathrm{nm}$, as in an odometer, and one gets the following relations

$$
V_{n m}=V_{n} \circ V_{m}=V_{m} \circ V_{n}, \quad F_{n m}=F_{n} \circ F_{m}=F_{m} \circ F_{n} .
$$

Next statement displays several equations connecting Frobenius and Verschiebung operators on $\mathbb{W}_{0}(\mathbb{S})$.

Proposition 2.4. The following relations hold with $m, n \in \mathbb{N}$

(1) $F_{n} \circ V_{n}(x)=n x$.

(2) $V_{n}\left(F_{n}(x) y\right)=x V_{n}(y)$. 


\section{Alain Connes and Caterina Consani}

(3) If $(m, n)=1, \quad V_{m} \circ F_{n}=F_{n} \circ V_{m}$.

(4) For $n \in \mathbb{N}, \quad V_{n}(x) V_{n}(y)=n V_{n}(x y)$.

Proof. The relations (1) and (4) are easily verified directly. The other two relations can be deduced from Theorem 2.3: the point being that a direct proof necessarily makes use of condition $(i)$ in Definition 2.2. In fact using the invariant $\tau$, we shall obtain the equalities

$$
\tau\left(V_{n}(x)\right)=\tilde{\rho}_{n}(\tau(x)), \quad \forall n \in \mathbb{N}, x \in \mathbb{W}_{0}(\mathbb{S}),
$$

where $\tilde{\rho}_{n}, n \in \mathbb{N}$, is defined by

$$
\tilde{\rho}_{n}: \mathbb{Z}[\mathbb{Q} / \mathbb{Z}] \rightarrow \mathbb{Z}[\mathbb{Q} / \mathbb{Z}], \quad \tilde{\rho}_{n}(e(\gamma))=\sum_{n \gamma^{\prime}=\gamma} e\left(\gamma^{\prime}\right) .
$$

The action of $\tau$ on $F_{n}$ is described by

$$
\tau\left(F_{n}(x)\right)=\sigma_{n}(\tau(x)), \quad \forall n \in \mathbb{N}, x \in \mathbb{W}_{0}(\mathbb{S}),
$$

where for each $n \in \mathbb{N}$ the group ring endomorphisms $\sigma_{n}$ is given by

$$
\sigma_{n}: \mathbb{Z}[\mathbb{Q} / \mathbb{Z}] \rightarrow \mathbb{Z}[\mathbb{Q} / \mathbb{Z}], \quad \sigma_{n}(e(\gamma))=e(n \gamma) .
$$

To prove (8) and (10) we claim that in terms of the Fourier transforms $\operatorname{gh}_{n}((E, T))=\tau \overline{(E, T)(n)}$ as in (5) one has

$$
\operatorname{gh}_{n}\left(V_{m}((E, T))\right)= \begin{cases}m \operatorname{gh}_{n / m}((E, T)) & \text { if } m \mid n \\ 0 & \text { otherwise }\end{cases}
$$

and

$$
\operatorname{gh}_{n}\left(\sigma_{m}((E, T))\right)=\operatorname{gh}_{n m}((E, T)) .
$$

Indeed, one verifies (12) and (13) directly using (5) and also easily checks that $\overline{\sigma_{m}(f)}(n)=\widehat{f}(n m)$. The Fourier transform of $\tilde{\rho}_{m}(e(\gamma))=\sum_{m \gamma^{\prime}=\gamma} e\left(\gamma^{\prime}\right)$ is

$$
\sum_{m \gamma^{\prime}=\gamma} \exp \left(2 \pi i n \gamma^{\prime}\right)= \begin{cases}m \exp (2 \pi i \gamma n / m) & \text { if } m \mid n \\ 0 & \text { otherwise. }\end{cases}
$$

This proves (8) and (10). The four equalities of Proposition 2.4 then follow from Proposition 5.1 of [10] that is also reported here below for completeness.

Proposition 2.5. The endomorphisms $\sigma_{n}$ and the maps $\tilde{\rho}_{m}$ fulfill the following relations

$$
\begin{gathered}
\sigma_{n m}=\sigma_{n} \sigma_{m}, \quad \tilde{\rho}_{m n}=\tilde{\rho}_{m} \tilde{\rho}_{n}, \quad \forall m, n \in \mathbb{N} \\
\tilde{\rho}_{m}\left(\sigma_{m}(x) y\right)=x \tilde{\rho}_{m}(y), \quad \forall x, y \in \mathbb{Z}[\mathbb{Q} / \mathbb{Z}] \\
\sigma_{c}\left(\tilde{\rho}_{b}(x)\right)=(b, c) \tilde{\rho}_{b^{\prime}}\left(\sigma_{c^{\prime}}(x)\right), \quad b^{\prime}=b /(b, c), \quad c^{\prime}=c /(b, c), \quad(b, c):=\operatorname{gcd}(b, c) .
\end{gathered}
$$

We note that taking $b=c=n$ in (16) gives

$$
\sigma_{n}\left(\tilde{\rho}_{n}(x)\right)=n x, \quad \forall x \in \mathbb{Z}[\mathbb{Q} / \mathbb{Z}] .
$$

On the other hand, if one takes $b=n$ and $c=m$ to be relatively prime one has

$$
\sigma_{n} \circ \tilde{\rho}_{m}=\tilde{\rho}_{m} \circ \sigma_{n}
$$




\section{$2.2 \mathbb{W}_{0}(\mathbb{S})$ and the integral BC-system}

We recall from [10] the main structure of the integral BC-system. The integral $B C$-algebra is the algebra $\mathcal{H}_{\mathbb{Z}}=\mathbb{Z}[\mathbb{Q} / \mathbb{Z}] \rtimes_{\tilde{\rho}} \mathbb{N}$ generated by the group ring $\mathbb{Z}[\mathbb{Q} / \mathbb{Z}]$, and by the elements $\tilde{\mu}_{n}$ and $\mu_{n}^{*}$, with $n \in \mathbb{N}$, which satisfy the relations

$$
\begin{aligned}
& \tilde{\mu}_{n m}=\tilde{\mu}_{n} \tilde{\mu}_{m}, \quad \forall n, m \in \mathbb{N} \\
& \mu_{n m}^{*}=\mu_{n}^{*} \mu_{m}^{*}, \quad \forall n, m \\
& \mu_{n}^{*} \tilde{\mu}_{n}=n \\
& \tilde{\mu}_{n} \mu_{m}^{*}=\mu_{m}^{*} \tilde{\mu}_{n} \quad(n, m)=1 .
\end{aligned}
$$

as well as the relations

$$
\begin{aligned}
& \tilde{\mu}_{n} x \mu_{n}^{*}=\tilde{\rho}_{n}(x) \\
& \mu_{n}^{*} x=\sigma_{n}(x) \mu_{n}^{*} \\
& x \tilde{\mu}_{n}=\tilde{\mu}_{n} \sigma_{n}(x),
\end{aligned}
$$

Notice that the equations (14), (15), (16) holding on $\mathcal{H}_{\mathbb{Z}}$ are the same as those fulfilled by the Frobenius endomorphisms $F_{n}$ and the Verschiebung maps $V_{n}$ on $\mathbb{W}_{0}(\mathbb{S})$ : see Proposition 2.4. More precisely, under the correspondences $\sigma_{n} \rightarrow F_{n}, \tilde{\rho}_{n} \rightarrow V_{n}$ the two equations of (7) correspond to (14), and the first three equations of Proposition 2.4 correspond respectively to (17), (15) and (18). These results evidently point out to the existence of a strong relation between the $(\lambda)$-ring $\mathbb{W}_{0}(\mathbb{S})$ and the ring $\mathbb{Z}[\mathbb{Q} / \mathbb{Z}]^{G}$ endowed with the aforementioned operators. This result is in agreement with the idea developed in [10] that the integral BC-system is the Witt ring of the algebraic closure of $\mathbb{F}_{1}$.

\section{$2.3 \lambda$-rings and the Arithmetic Site}

We refer to [3] for the notion of $\lambda$-ring: here we are only concerned with the case of special $\lambda$-rings in the terminology of op.cit. We recall that the Adams operations $\psi_{k}$ are endomorphisms defined explicitly in terms of the $\lambda$-operations $\lambda_{j}$. Thus for instance we have

$$
\begin{array}{ccc}
\psi_{1}= & \lambda_{1} \\
\psi_{2}= & \lambda_{1}^{2}-2 \lambda_{2} \\
\psi_{3}= & \lambda_{1}^{3}-3 \lambda_{2} \lambda_{1}+3 \lambda_{3} \\
\psi_{4}= & \lambda_{1}^{4}-4 \lambda_{2} \lambda_{1}^{2}+4 \lambda_{3} \lambda_{1}+2 \lambda_{2}^{2}-4 \lambda_{4}
\end{array}
$$

To obtain the general formula one uses (see op.cit. $\S 5$ ) the identity of formal power series

$$
t \partial_{t}(\lambda(t)) / \lambda(t)=\sum_{1}^{\infty}(-1)^{n} \psi_{n} t^{n}, \quad \lambda(t):=\sum_{0}^{\infty} \lambda_{n} t^{n} .
$$

Next, we observe that $\lambda$-rings belong naturally to the topos $\widehat{\mathbb{N}^{x}}$ that underlies the Arithmetic Site.

Lemma 2.6. Let $R$ be a $\lambda$-ring. Then the Adams operations $\psi_{n}$ turn $R$ into a sheaf of rings on the topos $\widehat{\mathbb{N}^{x}}$.

Proof. The semigroup $\mathbb{N}^{\times}$acts by endomorphisms on $R$ as follows [3] Proposition 5.2):

$$
(n, x) \mapsto \psi_{n}(x), \quad \forall x \in R, n \in \mathbb{N}^{\times},
$$

so that $R$, endowed with this action, becomes a sheaf of rings on the (presheaf) topos $\widehat{\mathbb{N}^{x}}$. 


\section{Alain Connes and Caterina Consani}

The definition of the Arithmetic Site was in fact motivated by the $\lambda$-operations in cyclic theory [31] and their role in the conceptual understanding of Serre's local factors of geometric L-functions at the archimedean places [9]. The whole construction relied on the cyclic category [6] and its epicyclic refinement [12]. It is a surprising fact that while the classifying spaces of these small categories are non-trivial [6], the classifying space of the simplicial category is indeed trivial even though the associated cohomology theory, the Hochschild cohomology, is non-trivial. It is exactly the theory of Grothendieck topoi that provides the best geometric framework to understand Hochschild and cyclic homologies together with the $\lambda$-operations. This is achieved using the topos associated to the cyclic category and its epicyclic refinement. The epicyclic topos $\left(\tilde{\Lambda}^{\mathrm{op}}\right)^{\wedge}$ is defined by considering the presheaf topos associated to the opposite of the epicyclic category $\tilde{\Lambda}$. In other words, this is the topos of covariant functors from $\tilde{\Lambda}$ to $\mathfrak{S e t s}$. This choice is dictated by the following natural construction. A commutative ring $R$ determines a covariant functor $R^{\#}: \mathfrak{F i n} \longrightarrow \mathfrak{A b}$ from the category of finite sets to abelian groups. This functor assigns to a finite set $J$ the tensor power $R^{\otimes J}=\bigotimes_{j \in J} R$ and to a map $\phi: J \rightarrow J^{\prime}$ the morphism of abelian groups

$$
R^{\#}(\phi): \bigotimes_{j \in J} R \rightarrow \bigotimes_{k \in J^{\prime}} R, \quad R^{\#}(\phi)\left(\otimes_{j \in J} x_{j}\right)=\otimes_{k \in J^{\prime}} y_{k}, \quad y_{k}=\prod_{\phi(j)=k} x_{j} .
$$

In [11] we explained in geometric terms that there is a natural covariant functor $\mathcal{P}: \tilde{\Lambda} \longrightarrow \mathfrak{F i n}$. In this construction, the involved geometry is projective geometry in characteristic 1 and $\tilde{\Lambda}$ is interpreted as a category of projective spaces with geometric morphisms. One lets $\mathbb{Z}_{\max }$ be the only semifield whose multiplicative group is infinite cyclic. One shows that the category $\tilde{\Lambda}$ is isomorphic to the category whose objects are the $\mathbb{Z}_{\text {max }}$-modules $\mathcal{E}_{n}$ whose underlying additive semigroup is $\mathbb{Z}_{\max }$ while the action of $\mathbb{Z}_{\max }$ is given by composing the multiplication with the Frobenius endomorphism $\operatorname{Fr}_{n}(x)=x^{n}$. The morphisms in the category are the projective transformations [11]. Then the functor $\mathcal{P}$ associates to a projective space its finite set of points, it is surjective on objects (up to isomorphism) and morphisms. Then, for any commutative ring $R$, the composite $R^{\#} \circ \mathcal{P}$ provides a covariant functor $R^{\natural}=R^{\#} \circ \mathcal{P}: \tilde{\Lambda} \longrightarrow \mathfrak{A b}$ i.e. a sheaf of abelian groups over the topos $\left(\tilde{\Lambda}^{\mathrm{op}}\right)^{\wedge}$. Both the cyclic homology of $R$ and its $\lambda$-operations are completely encoded by the associated sheaf $R^{\natural}$.

When one works with the category $\mathfrak{F i n}_{*}$ of finite pointed sets, instead of $\mathfrak{F i n}$, the corresponding structure involves, in place of $\tilde{\Lambda}$ the semi-direct product category $\Delta^{\mathrm{op}} \ltimes \mathbb{N}^{\times}$. Here we view $\Delta^{\mathrm{op}}$ as the category of finite intervals $[n]^{*}:=\{0, \ldots, n+1\}$ with $n+2$ elements (i.e. $n$ elements besides the smallest and largest) with morphisms preserving the smallest and largest elements. The action $\operatorname{Sd}_{k}^{*}$ of $k \in \mathbb{N}^{\times}$on $\Delta^{\mathrm{op}}$ is understood as the concatenation of $k$ copies of the interval (see op.cit. Lemma 2.2), it is the dual of the edgewise subdivision. One has a natural covariant functor $\mathcal{F}: \Delta^{\mathrm{op}} \longrightarrow \Gamma^{\mathrm{op}}$ that associates to an interval $I=\{b, \ldots, t\}$ the pointed set $I_{\star}=I / \sim$ with base point the class of $b \sim t$ obtained by identifying the smallest and largest elements of $I$. To any morphism of intervals $f: I \rightarrow J$ corresponds the quotient map $f_{\star}$ which preserves the base point. The semi-direct product category $\Delta^{\mathrm{op}} \ltimes \mathbb{N}^{\times}$has the same objects as $\Delta^{\mathrm{op}}$ and new morphisms $\pi_{n}^{k}: \operatorname{Sd}_{k}^{*}\left([n]^{*}\right) \rightarrow[n]^{*}$. The functor $\mathcal{F}$ can be extended to $\Delta^{\mathrm{op}} \ltimes \mathbb{N}^{\times}$as follows (see op.cit. Proposition 2.8)

Proposition 2.7. For any $n \geq 0, k \in \mathbb{N}^{\times}$, let $\left(\pi_{n}^{k}\right)_{*}: \mathcal{F}\left(\operatorname{Sd}_{k}^{*}\left([n]^{*}\right)\right) \rightarrow \mathcal{F}\left([n]^{*}\right)$ be given by the residue modulo $n+1$. Then the extension of the functor $\mathcal{F}$ on morphisms given by

$$
\phi=\pi_{n}^{k} \circ \alpha \mapsto \phi_{*}:=\left(\pi_{n}^{k}\right)_{*} \circ \alpha_{*}
$$

determines a functor $\mathcal{F}: \Delta^{\mathrm{op}} \ltimes \mathbb{N}^{\times} \longrightarrow \Gamma^{\mathrm{op}}$ which is surjective on objects and morphisms. 
The construction of the $\lambda$-operations on Hochschild homology of a commutative ring [31], only uses the functor $R^{\#} \circ \mathcal{F}: \Delta^{\mathrm{op}} \ltimes \mathbb{N}^{\times} \longrightarrow \mathfrak{A} \mathfrak{b}$. In view of the fact that the presheaf topos $\Gamma^{\wedge}$ of covariant functors $\Gamma^{\mathrm{op}} \longrightarrow \mathfrak{S e t s}$ plays a key role in defining the category $\mathbb{S}$ - Mod of $\mathbb{S}$-modules, there is the need to clarify the relations between the various candidates for the geometric counterpart of the "absolute point", given by the following topoi:

(i) The Arithmetic Site $\widehat{\mathbb{N}^{x}}$.

(ii) The topos $\Gamma^{\wedge}$ of covariant functors $\Gamma^{\mathrm{op}} \longrightarrow$ Sets.

(iii) The topos of covariant functors $\Delta^{\mathrm{op}} \ltimes \mathbb{N}^{\times} \longrightarrow$ Sets.

(iv) The epicyclic topos $\left(\tilde{\Lambda}^{\mathrm{op}}\right)^{\wedge}$.

\section{Probabilistic interpretation of the $\theta$-dimension}

In this section we give a probabilistic interpretation of the $\theta$-dimension of an Arakelov divisors $D$ on $\overline{\operatorname{Spec} \mathbb{Z}}$. Given an euclidean lattice $\bar{L}:=(L,\|\|$.$) its \theta$-invariant is defined (see $[5,37])$ by

$$
h_{\theta}^{0}(\bar{L}):=\log \sum_{v \in L} \exp \left(-\pi\|v\|^{2}\right) .
$$

We refer to [5] for the extension of the definition and properties of the $\theta$-invariant to the infinite dimensional case and its interpretation using the thermodynamic formalism. Here, we provide a different interpretation of the same invariant based on a probabilistic way of counting naively the solutions involving a one-dimensional complex vector space as customary in Arakelov geometry.

\subsection{The $\mathbb{S}$-module $E_{\xi}$}

Let $E$ be a one dimensional complex vector space. Given a vector $\xi \in E$ we define the $\mathbb{S}$-module

$$
E_{\xi}\left(k_{+}\right):=\left\{\phi \in E^{k} \mid \sum_{x \in k_{+}}\|\phi(x)\| \leq\|\xi\|\right\} .
$$

The above definition is independent of the choice of the norm \|\| on $E$. Given a rank one discrete subgroup $L \subset E$ of the additive group of $E$ one can roughly measure the size of a vector $\xi \in E$ by comparing it with the unit of length provided by $L$. This yields the quantity

$$
[\xi / L]:=\#\left(H L \cap E_{\xi}\right)\left(1_{+}\right),
$$

where $H L$ denotes the Eilenberg-MacLane $\mathbb{S}$-module associated to the abelian group $L$ (see [23] Example 2.1.2.1). One has $H L\left(k_{+}\right):=L^{k}$ and the functoriality is that of $L$-valued divisors on finite pointed sets. Note that this invariant of the pair $(L \subset E, \xi)$ does not depend on the choice of the norm on $E$.

\subsection{The Gaussian measure}

Next we recall the well-known characterization of the Gaussian measure

Lemma 3.1. Let $\mathcal{H}$ be a one dimensional complex Hilbert space. There exists a unique probability measure $d p$ on $\mathcal{H}$ fulfilling the following properties

- $d p$ is rotation invariant.

- Two real linear forms which are orthogonal are independent random variables.

- The mean value of the norm is $\frac{1}{2}$. 


\section{Alain Connes and Caterina Consani}

Proof. We can assume that $\mathcal{H}=\mathbb{C}$ with the absolute value as the norm. The measure $d p(z):=$ $\exp \left(-\pi|z|^{2}\right) d x d y$, with $z=x+i y$ fulfills the required conditions. Note that the first two requirements on $d p$ do not use the norm and fix the density of the measure to be a Gaussian $a \exp \left(-\pi a|z|^{2}\right)$. Since the expectation value of the norm on this probability measure is

$$
a \int_{-\infty}^{\infty} \int_{-\infty}^{\infty} \sqrt{x^{2}+y^{2}} \exp \left(-\pi a x^{2}-\pi a y^{2}\right) d x d y=\frac{1}{2 a^{1 / 2}}
$$

the last condition fixes uniquely the normalization constant $a=1$ and hence the measure.

\subsection{Probabilistic formula for $\exp \left(h_{\theta}^{0}(D)\right)$}

To an Arakelov divisor $D=\sum u_{j}\left\{p_{j}\right\}+u\{\infty\}$ on $\overline{\mathrm{Spec} \mathbb{Z}}$ is associated the euclidean lattice

$$
H^{0}(D):=\left(H^{0}\left(\operatorname{Spec} \mathbb{Z}, \mathcal{O}\left(D_{\text {finite }}\right)\right), e^{-u}\|\|\right)
$$

where the invertible sheaf $\mathcal{O}\left(D_{\text {finite }}\right)$ on $\operatorname{Spec} \mathbb{Z}$ is viewed as a subsheaf of the constant sheaf $\mathbb{Q}$ and the norm \|\| is the restriction of the euclidean norm on $\mathbb{Q}$.

Definition 3.2. Let $D$ be an Arakelov divisor on $\overline{\mathrm{Spec} \mathbb{Z}}$. We let

$$
H_{\mathbb{C}}^{0}(D)=(L \subset E,\|\|),
$$

where $E$ is the one dimensional Hilbert space $E=\mathbb{C}$ with norm $e^{-u}\|\|$ and $L \subset E$ is the rank one discrete subgroup $L=H^{0}\left(\operatorname{Spec} \mathbb{Z}, \mathcal{O}\left(D_{\text {finite }}\right) \subset \mathbb{Q} \subset \mathbb{C}\right.$ of formula $(25)$.

The role of the complexification $H_{\mathbb{C}}^{0}(D)=(L \subset E,\|\|)$ is exhibited by the following result

Theorem 3.3. Let $d p$ be the unique measure on $E$ defined in Lemma 3.1. One has

$$
\exp \left(h_{\theta}^{0}(D)\right)=\int[\xi / L] d p(\xi) .
$$

Proof. The pair $H_{\mathbb{C}}^{0}(D)$ associated to $D$ by Definition 3.2 is unchanged, up to isomorphism, if one replaces $D$ with $D+(q)$ i.e. by adding a principal divisor. Thus we can assume that $D=u\{\infty\}$, with $u=\operatorname{deg}(D) \in \mathbb{R}$. Then the pair $H_{\mathbb{C}}^{0}(D)=(L \subset E,\|\|)$ is the same as the pair $(\mathbb{Z} \subset E,\|\|)$, where $E$ is the one dimensional Hilbert space $E=\mathbb{C}$ with norm $\|z\|:=e^{-u}|z|$. The measure $d p$ on $E$ as in Lemma 3.1 is then (since $e^{-u} \frac{1}{2 a^{1 / 2}}=\frac{1}{2}$ )

$$
d p(z):=a \exp \left(-\pi a|z|^{2}\right) d x d y, \quad z=x+i y, \quad a=e^{-2 u} .
$$

For $z \in E=\mathbb{C}$ one has $[z / \mathbb{Z}]=1+2 \operatorname{IntegerPart}(|z|)$, thus the right hand side of $(26)$ becomes

$$
\int[\xi / L] d p(\xi)=a \int(1+2 \operatorname{IntegerPart}(|z|)) \exp \left(-\pi a|z|^{2}\right) d x d y, \quad a=e^{-2 u} .
$$

Let $\chi:=1_{[-1,1]}$ be the characteristic function of the unit interval, one has

$$
\operatorname{IntegerPart}(|z|)=\sum_{\mathbb{N}^{x}} \chi\left(\frac{n}{|z|}\right)
$$

and by applying Fubini's Theorem we obtain

$$
\int[\xi / L] d p(\xi)=1+2 a \sum_{\mathbb{N}^{\times}} \int \chi\left(\frac{n}{|z|}\right) \exp \left(-\pi a|z|^{2}\right) d x d y .
$$

One has

$a \int \chi\left(\frac{n}{|z|}\right) \exp \left(-\pi a|z|^{2}\right) d x d y=a \int_{|z| \geq n} \exp \left(-\pi a|z|^{2}\right) d x d y=2 \pi a \int_{n}^{\infty} \exp \left(-\pi a \rho^{2}\right) \rho d \rho=\exp \left(-\pi a n^{2}\right)$ 
so that

$$
\int[\xi / L] d p(\xi)=1+2 \sum_{\mathbb{N}^{x}} \exp \left(-\pi a n^{2}\right) .
$$

This gives the required formula using (22) since, as $a=e^{-2 u}, a n^{2}$ is the square of the norm of the integer $n$ using (25).

\section{4. $H^{1}(\overline{\operatorname{Spec} \mathbb{Z}}, \mathcal{O}(D))$ and the Dold-Kan correspondence for $\mathbb{S}$-modules}

In this section we apply the analogue of the Dold-Kan correspondence in the framework of $\mathbb{S}$ modules to construct a $\Gamma$-space $\mathbf{H}(D)$ associated to an Arakelov divisor $D$ on $\overline{\operatorname{Spec} \mathbb{Z}}$. $\mathbf{H}(D)$ is the homological incarnation of the Riemann-Roch problem for $D$ and is built from a natural morphism of $\mathbb{S}$-modules canonically associated to the divisor $D$, as explained in $\S 4.2$.

\subsection{The structure sheaf of $\overline{\mathrm{Spec} \mathbb{Z}}$}

We briefly recall the definition of the structure sheaf of $\mathbb{S}$-algebras on the Arakelov compactification Spec $\mathbb{Z} \subset \overline{\text { Spec } \mathbb{Z}}$ as introduced in [19]. Here, we also show that the construction extends to a one parameter deformation by implementing a real parameter $0<\alpha \leq 1$ in the key proposition of op.cit. that provides the extension of the structure sheaf of $S p e c \mathbb{Z}$ at the real archimedean place and introduces the module $\mathcal{O}(D)$ attached to a divisor $D$.

Proposition 4.1. Let $0<\alpha \leq 1$ be a real number and let $R$ be a semiring.

(i) Let \|\| be a sub-multiplicative seminorm on $R$. Then $H R$ is naturally endowed with a structure of $\mathbb{S}$-subalgebra $\|H R\|_{1}^{\alpha} \subset H R$ defined as follows

$$
\|H R\|_{1}^{\alpha}: \Gamma^{\mathrm{op}} \longrightarrow \mathfrak{S e t s}_{*} \quad\|H R\|_{1}^{\alpha}(F):=\left\{\phi \in H R(F) \mid \sum_{F \backslash\{*\}}\|\phi(x)\|^{\alpha} \leq 1\right\} .
$$

(ii) Let $E$ be an $R$-semimodule and \|\| a seminorm on $E$ such that $\|a \xi\| \leq\|a\|\|\xi\|, \forall a \in R, \forall \xi \in E$. Then for any $\lambda \in \mathbb{R}_{+}$the following formula defines a module $\|H E\|_{\lambda}^{\alpha}$ over $\|H R\|_{1}^{\alpha}$

$$
\|H E\|_{\lambda}^{\alpha}: \Gamma^{\mathrm{op}} \longrightarrow \mathfrak{S e t s}_{*} \quad\|H E\|_{\lambda}^{\alpha}(F):=\left\{\phi \in H E(F) \mid \sum_{F \backslash\{*\}}\|\phi(x)\|^{\alpha} \leq \lambda\right\} .
$$

Proof. One needs to show that the maps $H E(f)(\phi)(y):=\sum_{x \mid f(x)=y} \phi(x)$ are compatible with the conditions $\sum_{X \backslash\{*\}}\|\phi(x)\|^{\alpha} \leq \lambda$. For $\alpha \leq 1$ and for any positive real numbers $a, b$ one has the inequality $(a+b)^{\alpha} \leq a^{\alpha}+b^{\alpha}$. Thus, since the map $a \mapsto a^{\alpha}$ is increasing, we obtain, using the inequality

$$
\left\|\sum_{x \mid f(x)=y} \phi(x)\right\| \leq \sum_{x \mid f(x)=y}\|\phi(x)\|
$$

that

$$
\left\|\sum_{x \mid f(x)=y} \phi(x)\right\|^{\alpha} \leq\left(\sum_{x \mid f(x)=y}\|\phi(x)\|\right)^{\alpha} \leq \sum_{x \mid f(x)=y}\|\phi(x)\|^{\alpha} .
$$

One obtains, as required

$$
\sum_{Y \backslash\{*\}}\|H E(f)(\phi)(y)\|^{\alpha}=\sum_{Y \backslash\{*\}}\left\|\sum_{x \mid f(x)=y} \phi(x)\right\|^{\alpha} \leq \sum_{X \backslash\{*\}}\|\phi(x)\|^{\alpha} \leq \lambda .
$$

The sub-multiplicativity of the norm entails the compatibility with the product. 


\section{Alain Connes and Caterina Consani}

REMARK 4.2. For $\alpha>1$ it is no longer true that (28) defines a sub $\Gamma$-set of $H E$, since taking $\phi(x)=\xi \neq 0$ for all $x$, one has when $\#\{x \mid f(x)=y\}=n>1$,

$$
\left\|\sum_{x \mid f(x)=y} \phi(x)\right\|^{\alpha}=n^{\alpha}\|\xi\|^{\alpha}>\sum_{x \mid f(x)=y}\|\phi(x)\|^{\alpha}=n\|\xi\|^{\alpha} .
$$

\subsection{The morphism $\phi$}

For a function field $\mathbb{K}$ with field of constants $k, \mathrm{~A}$. Weil gave a proof of the Riemann-Roch formula using the topological ring of adeles and Pontrjagin duality. The proof provides, in particular, an adelic interpretation of $H^{1}(D)$, for divisors $D$ of $\mathbb{K}$. For every place $v \in \Sigma_{\mathbb{K}}$ of $\mathbb{K}$, the local field $\mathbb{K}_{v}$ admits a canonical ultrametric norm ||$_{v}: \mathbb{K}_{v}^{\times} \rightarrow \mathbb{R}_{+}^{*}$ : we let $\operatorname{Mod}\left(\mathbb{K}_{v}\right)$ the range of ||$_{v}$. Then, one can view a divisor of $\mathbb{K}$ as a map $D: \Sigma_{\mathbb{K}} \rightarrow \mathbb{R}_{+}^{*}$ on the set $\Sigma_{\mathbb{K}}$ of places of $\mathbb{K}$ such that $D(v) \in \operatorname{Mod}\left(\mathbb{K}_{v}\right), \forall v \in \Sigma_{\mathbb{K}}$ and fulfilling the condition $D(v)=1$ for all but finitely many places. A divisor $D$ defines a compact open subgroup $\mathcal{O}(D)$ of the additive group $\mathbb{A}_{\mathbb{K}}$ of adeles of $\mathbb{K}$ by setting

$$
\mathcal{O}(D):=\left\{a=\left.\left(a_{v}\right) \in \mathbb{A}_{\mathbb{K}}|| a_{v}\right|_{v} \leq D(v), \forall v \in \Sigma_{\mathbb{K}}\right\} .
$$

By implementing the diagonal embedding of $\mathbb{K}$ in $\mathbb{A}_{\mathbb{K}}$, one defines the morphism

$$
\psi: \mathbb{K} \oplus \mathcal{O}(D) \rightarrow \mathbb{A}_{\mathbb{K}}, \psi(x, y):=x+y \in \mathbb{A}_{\mathbb{K}}, \quad \forall x \in \mathbb{K}, y \in \mathcal{O}(D) .
$$

The kernel and cokernel of $\psi$ define respectively the following two natural $k$-vector spaces

$$
H^{0}(D):=\mathcal{O}(D) \cap \mathbb{K}, \quad H^{1}(D):=\mathbb{A}_{\mathbb{K}} /(\mathbb{K}+\mathcal{O}(D)) .
$$

These definitions use in a crucial way the ultrametric property of the local norm ||$_{v}$ and the existence of the field of constants.

For number fields, the ultrametric property of the local norm fails at the archimedean places hence the compact subset of adeles as in (29) is no longer an additive group. On the other hand, the map $\psi$ as in (30) still retains a meaning. We consider the simplest case of the field $\mathbb{Q}$. To assign a map $D: \Sigma_{\mathbb{Q}} \rightarrow \mathbb{R}_{+}^{*}$ with $D(v) \in \operatorname{Mod}\left(\mathbb{Q}_{v}\right), \forall v \in \Sigma_{\mathbb{Q}}$, and such that $D(v)=1$ for all but finitely many places, is equivalent to define an Arakelov divisor $D=\sum a_{j}\left\{p_{j}\right\}+a\{\infty\}$ on $\overline{\operatorname{Spec} \mathbb{Z}}$. Indeed, one sets $D\left(p_{j}\right):=p_{j}^{a_{j}}, D(v)=1$ for any finite place $v \neq p_{j} \forall j$, and $D(\infty)=e^{a}$. Then $(29)$ defines a compact subset $\mathcal{O}(D) \subset \mathbb{A}_{\mathbb{Q}}$. The analogue of $(30)$ is now the map

$$
\psi: \mathbb{Q} \times \mathcal{O}(D) \rightarrow \mathbb{A}_{\mathbb{Q}}, \psi(x, y):=x+y \in \mathbb{A}_{\mathbb{Q}}, \quad \forall x \in \mathbb{Q}, y \in \mathcal{O}(D)
$$

We let $j: \mathbb{A}_{\mathbb{Q}}^{\mathrm{f}} \rightarrow \mathbb{A}_{\mathbb{Q}}, j(x):=(x, 0)$, be the embedding of finite adeles in adeles. Using the ultrametric property of the local norms at the finite places one sees that $\mathcal{O}(D)^{\mathrm{f}}:=\mathcal{O}(D) \cap j\left(\mathbb{A}_{\mathbb{Q}}^{\mathrm{f}}\right)$ is a compact subgroup of the additive group $\mathbb{A}_{\mathbb{Q}}$. Let $G=\mathbb{Q} \times \mathcal{O}(D)^{\mathrm{f}}$ : one has $\mathcal{O}(D)^{\mathrm{f}} \cap \mathbb{Q}=\{0\}$ since all elements of $\mathcal{O}(D)^{\mathrm{f}}$ have archimedean component $=0$. Thus, the restriction of the map $\psi$ as in (32) to $G$ determines an isomorphism of $G$ with the subgroup $\psi(G)=\mathbb{Q}+\mathcal{O}(D)^{\mathrm{f}}$ of $\mathbb{A}_{\mathbb{Q}}$. Note that $\psi(G)$ is closed in $\mathbb{A}_{\mathbb{Q}}$, since $\mathbb{Q}$ is discrete (hence closed) and $\mathcal{O}(D)^{\mathrm{f}}$ is compact. It follows that $G$ plays no role in the definition of the kernel and cokernel of $\psi$ and one can reduce the analysis of this map by moding out $G$ as follows

Lemma 4.3. After quotienting both sides of (32) by $G=\mathbb{Q} \times \mathcal{O}(D)^{\mathrm{f}}$, the map $\psi$ becomes

$$
\phi:\left[-e^{a}, e^{a}\right] \rightarrow \mathbb{R} / L, \quad \phi(x):=x L \in \mathbb{R} / L, \quad \forall x \in\left[-e^{a}, e^{a}\right] \subset \mathbb{R},
$$

where $L \subset \mathbb{Q} \subset \mathbb{R}$ is the lattice

$$
L=H^{0}\left(\operatorname{Spec} \mathbb{Z}, \mathcal{O}\left(D_{\text {finite }}\right)\right):=\left\{\left.q \in \mathbb{Q}|| q\right|_{v} \leq D_{v}, \quad \forall v \neq \infty\right\} .
$$


Proof. We identify (set-theoretically) the adeles $\mathbb{A}_{\mathbb{Q}}$ with the product $\mathbb{A}_{\mathbb{Q}}^{f} \times \mathbb{R}$ endowed with projection morphisms $p_{\mathrm{f}}: \mathbb{A}_{\mathbb{Q}} \rightarrow \mathbb{A}_{\mathbb{Q}}^{\mathrm{f}}$ and $p_{\infty}: \mathbb{A}_{\mathbb{Q}} \rightarrow \mathbb{R}$. The subgroup $p_{\mathrm{f}}(\mathbb{Q}) \subset \mathbb{A}_{\mathbb{Q}}^{\mathrm{f}}$ is dense and the subgroup $p_{\mathrm{f}}\left(\mathcal{O}(D)^{\mathrm{f}}\right) \subset \mathbb{A}_{\mathbb{Q}}^{\mathrm{f}}$ is open. Hence $p_{\mathrm{f}}(\psi(G))=\mathbb{A}_{\mathbb{Q}}^{\mathrm{f}}$. The kernel of the restriction of $p_{\mathrm{f}} \circ \psi$ to $G$ is the group of pairs $(q, a) \in \mathbb{Q} \times \mathcal{O}(D)^{\mathrm{f}}$ such that $p_{\mathrm{f}}(q)+p_{\mathrm{f}}(a)=0$. Such pairs are determined by the value of $q$ and this detects the lattice

$$
\left\{\left.q \in \mathbb{Q}|| q\right|_{v} \leq D_{v}, \quad \forall v \neq \infty\right\}=L .
$$

Thus one obtains an isomorphism of groups $p: \mathbb{A}_{\mathbb{Q}} / \psi(G) \stackrel{\sim}{\rightarrow} \mathbb{R} / L$ by taking the restriction of the projection $p_{\infty}$ to the kernel of $p_{\mathrm{f}}$. By construction one has $\mathbb{Q} \times \mathcal{O}(D) \simeq G \times\left[-e^{a}, e^{a}\right]$, where the quotient by $G$ is identified with $\{0\} \times\left[-e^{a}, e^{a}\right]$, while the map $\psi$ of (32) is replaced by the map $\phi$ of (33).

We can upgrade the morphism $\phi$ to a morphism of $\mathbb{S}$-modules. Let $L \subset \mathbb{R}$ be a lattice, $q: \mathbb{R} \rightarrow \mathbb{R} / L$ the quotient morphism of abelian groups, and $H q: H \mathbb{R} \rightarrow H(\mathbb{R} / L)$ the associated morphism of $\mathbb{S}$-modules. For $\lambda>0$, and using the notations as in $\S 4.1$, one has a natural inclusion of $\mathbb{S}$-modules $\|H \mathbb{R}\|_{\lambda} \stackrel{\iota}{\rightarrow} H \mathbb{R}$. Thus one derives the following morphism of $\mathbb{S}$-modules which coincides with the above $\phi$ at level 1

$$
\phi:\|H \mathbb{R}\|_{\lambda} \rightarrow H(\mathbb{R} / L), \quad \phi:=H q \circ \iota .
$$

\subsection{Dold-Kan correspondence for $\phi: A \rightarrow B$}

Let $\phi: A \rightarrow B$ be a morphism of abelian groups. We describe the simplicial abelian group $\mathcal{A}$ associated, by the Dold-Kan correspondence, to the following complex $\mathfrak{C}=\left\{C_{n}, \phi_{n}\right\}$ indexed in non-negative degrees, and defined as follows

$$
\mathfrak{C}=\left\{C_{1} \stackrel{\phi_{1}}{\rightarrow} C_{0}\right\} ; \quad C_{0}=B, C_{1}=A, \quad \phi_{1}(x):=\phi(x), \quad \forall x \in A .
$$

The goal of this section is to obtain a description of $\mathcal{A}$ that is easy to transpose when the above morphism $\phi$ is replaced by the morphism of $\mathbb{S}$-modules as in (35).

We use the following definition of the simplicial abelian group $\mathcal{A}$ associated, by the Dold-Kan correspondence, to a complex $C$ of abelian groups

$$
\mathcal{A}_{n}:=\operatorname{Hom}_{\mathrm{Ch}_{+}}\left(N\left(H \mathbb{Z} \circ \Delta^{n}\right), C\right) \quad \forall n \geq 0,
$$

where $H \mathbb{Z} \circ \Delta^{n}$ is the free simplicial abelian group associated to the $n$-simplex (i.e. the simplicial set $\operatorname{Hom}(\bullet,[n]))$, and $N$ denotes the normalized Moore complex of a simplicial abelian group. There is a concrete description of $\mathcal{A}_{n}$ given as follows (see [25] §III.2, Prop. 2.2)

$$
\mathcal{A}_{n}=\bigoplus_{\mathcal{F}(n, k)} C_{k}, \quad \mathcal{F}(n, k):=\left\{\sigma \in \operatorname{Hom}_{\Delta}([n],[k]) \mid \sigma([n])=[k]\right\},
$$

here the direct sum repeats the term $C_{k}$ of the chain complex as many times as the number of elements of the set $\mathcal{F}(n, k)$ of surjective morphisms $\sigma \in \mathrm{Hom}_{\Delta}([n],[k])$. In the simple case of the short complex $\mathfrak{C}$ in (36), the allowed values of $k$ are $k=0,1$. In this case, the set $\mathcal{F}(n, 0)$ is reduced to a single element: $\operatorname{Hom}_{\Delta}([n],[0])=: \Delta_{n}^{0}$. Next, we determine $\mathcal{F}(n, 1)$. An element $\xi \in \operatorname{Hom}_{\Delta}([n],[1])=\Delta_{n}^{1}$ is characterized by $\xi^{-1}(\{1\})$ that is an hereditary subset of $[n]$. Thus the vertices in $\Delta_{n}^{1}$ are labelled as

$$
\xi_{j}, 0 \leq j \leq n+1, \xi_{j}(k)=1 \Longleftrightarrow k \geq j .
$$

For each integer $n \geq 0$ the finite set $F(n):=\mathcal{F}(n, 1)$ of surjective elements $\xi \in \Delta_{n}^{1}$ excludes $\xi_{0}$ and $\xi_{n+1}$, thus $F(n)=\left\{\xi_{j} \mid j \in\{1, \ldots, n\}\right\}$ has $n$ elements. In the simple case of the complex $\mathfrak{C}$ one 


\section{Alain Connes and Caterina Consani}

can rewrite (37) as

$$
\mathcal{A}_{n}=H B\left(1_{+}\right) \oplus H A\left(F(n)_{+}\right) .
$$

For the description of the simplicial structure, namely the definition for each $\theta \in \operatorname{Hom}_{\Delta}([m],[n])$ of a map of sets $\mathcal{A}(\theta): \mathcal{A}_{n} \rightarrow \mathcal{A}_{m}$ we follow again [25] §III.2, p. 160-161. Let, as in $\S 2.1$, $\mathfrak{S e t s}_{2, *}$ be the category of pairs of pointed sets $(X, Y)$, with $X \supset Y$ where morphisms are maps of pairs of pointed sets, and $\gamma: \mathfrak{S e t s}_{2, *} \longrightarrow \mathfrak{S e t s}_{*}$ the functor $(X, Y) \rightarrow X / Y$ of collapsing $Y$ to the base point. One has the following

Proposition 4.4. Let $\phi: A \rightarrow B$ be a morphism of abelian groups. The following setting defines a covariant functor

$$
H_{\phi}: \mathfrak{S e t s}_{2, *} \longrightarrow \mathfrak{A} \mathfrak{b}, \quad H_{\phi}(X, Y):=H B(Y) \times H A(X / Y) .
$$

For a morphism $f:(X, Y) \rightarrow\left(X^{\prime}, Y^{\prime}\right)$ of pairs of pointed sets, lets, with $\psi=\left(\psi_{Y}, \psi_{X / Y}\right) \in$ $H_{\phi}(X, Y), y^{\prime} \neq *$,

$$
H_{\phi}(f)(\psi):=\left(\psi_{Y^{\prime}}, H A(f)\left(\psi_{X / Y}\right)\right), \psi_{Y^{\prime}}\left(y^{\prime}\right):=H B(f)\left(\psi_{Y}\right)\left(y^{\prime}\right)+\sum_{x \in X \backslash Y, f(x)=y^{\prime}} \phi\left(\psi_{X / Y}(x)\right) .
$$

Proof. The sum in (41) is well defined and determines an element of $B$ for each $y^{\prime} \in Y^{\prime}, y^{\prime} \neq *$. By construction $H_{\phi}(f)$ is a morphism of abelian groups. Let $g:\left(X^{\prime}, Y^{\prime}\right) \rightarrow\left(X^{\prime \prime}, Y^{\prime \prime}\right)$ be a morphism in $\mathfrak{S e t s}_{2, *}$, and $h=g \circ f$. Let $y^{\prime \prime} \in Y^{\prime \prime}, y^{\prime \prime} \neq *$. We show that $H_{\phi}(h)=H_{\phi}(g) \circ H_{\phi}(f)$. We view the elements $\psi=\left(\psi_{Y}, \psi_{X / Y}\right) \in H_{\phi}(X, Y)$ as functions, with $\psi(x) \in A \cup B$, for $x \in X$ such that

$$
\psi(x) \in A, \quad \forall x \in X \backslash Y, \text { and } \psi(y) \in B, \quad \forall y \in Y, y \neq *, \psi(*)=0 .
$$

We define $p: A \cup B \rightarrow B$ to be equal to $\phi$ on $A$ and the identity on $B$. Then one has

$$
p\left(\sum a_{j}\right)=\sum p\left(a_{j}\right), \quad \forall a_{j} \in A, \quad p\left(\sum p\left(b_{j}\right)\right)=\sum p\left(b_{j}\right), \quad \forall b_{j} \in A \cup B .
$$

It follows from (41) that

$$
H_{\phi}(f)(\psi)(y)= \begin{cases}\sum_{x \in X, f(x)=y} \psi(x) & \text { if } y \notin Y^{\prime} \\ \sum_{x \in X, f(x)=y} p(\psi(x)) & \text { if } y \in Y^{\prime}, y \neq *\end{cases}
$$

By applying the functoriality of $H A$ and of the collapsing functor $(X, Y) \longrightarrow X / Y$, one checks that the equality $H_{\phi}(h)(\psi)=H_{\phi}(g)\left(H_{\phi}(f)(\psi)\right)$ holds for the evaluation on $x \in X \backslash Y$. Next, we take $y \in Y, y \neq *$ and evaluate both sides

$$
\begin{gathered}
H_{\phi}(h)(\psi)(y)=\sum_{x \in X, h(x)=y} p(\psi(x))=\sum_{z \in X, g(z)=y} \sum_{x \in X, f(x)=z} p(\psi(x)) \\
H_{\phi}(g)\left(H_{\phi}(f)(\psi)\right)(y)=\sum_{z \in X, g(z)=y} p\left(H_{\phi}(f)(\psi)(z)\right) .
\end{gathered}
$$

Using (43) and (44) one obtains

$$
p\left(H_{\phi}(f)(\psi)(z)\right)=\sum_{x \in X, f(x)=z} p(\psi(x)) .
$$

This argument shows that $H_{\phi}(h)=H_{\phi}(g) \circ H_{\phi}(f)$, thus proving the required functoriality of $H_{\phi}$.

As already mentioned in $\S 2.3$, the category $\Delta^{\mathrm{op}}$ is naturally isomorphic (hence identified) to (a skeleton of) the category of finite intervals, where by an interval we mean a totally ordered 
set with a smallest element distinct from a largest element. A morphism of intervals is a nondecreasing map that preserves the smallest and largest elements. The canonical contravariant functor $\Delta \longrightarrow \Delta^{\text {op }}$ that identifies the opposite category of $\Delta$ with $\Delta^{\mathrm{op}}$ (described by intervals as above) maps the finite ordinal $[n]=\{0,1, \ldots, n\}$ to the interval $[n]^{*}:=\{0, \ldots, n+1\}$. The dual of $\theta \in \operatorname{Hom}_{\Delta}([n],[m])$ is

$$
\left.\theta^{*} \in \operatorname{Hom}_{\Delta^{\mathrm{op}}}\left([m]^{*},[n]^{*}\right)\right), \quad j \leq \theta(i) \Longleftrightarrow \theta^{*}(j) \leq i .
$$

The bijection of sets $\operatorname{Hom}_{\Delta}([n],[m]) \stackrel{\theta \mapsto \theta^{*}}{\longrightarrow} \operatorname{Hom}_{\Delta^{\mathrm{op}}}\left([m]^{*},[n]^{*}\right)$ implements the identification

$$
\Delta_{n}^{1}=\operatorname{Hom}_{\Delta}([n],[1]) \stackrel{\sim}{\rightarrow} \operatorname{Hom}_{\Delta \mathrm{op}}\left([1]^{*},[n]^{*}\right)=[n]^{*}
$$

and one easily checks that under this identification the map $\xi_{j}$ of (38) corresponds to $j \in[n]^{*}=$ $\{0, \ldots, n+1\}$.

We let $\partial: \Delta^{\text {op }} \longrightarrow \mathfrak{S e t s}_{2, *}$ be the functor that replaces an interval $I$ by the pair $\partial I:=(X, Y)$, where $X$ is the set $I$ pointed by its smallest element, and $Y \subset X$ is the subset formed by the smallest and largest elements of $I$.

Next proposition describes the simplicial abelian group that corresponds to the complex $\mathfrak{C}$, by the Dold-Kan correspondence

Proposition 4.5. Let $\phi: A \rightarrow B$ be a morphism of abelian groups. Then, the simplicial abelian group associated by the Dold-Kan correspondence to the short complex $\mathfrak{C}$ in (36) is canonically isomorphic to the composite functor $H_{\phi} \circ \partial: \Delta^{\mathrm{op}} \longrightarrow \mathfrak{A} \mathfrak{b}$ with $H_{\phi}: \mathfrak{S e t s}_{2, *} \longrightarrow \mathfrak{A b}$ as in Proposition 4.4.

Proof. For $n \in \mathbb{N}$, the pair $\partial[n]^{*}$ is identified, using (46) and (38), to the pair $(X, Y)$ with

$$
X:=\Delta_{n}^{1}=\operatorname{Hom}_{\Delta}([n],[1]), \quad Y:=\left\{\xi_{0}, \xi_{n+1}\right\},
$$

with base point $\xi_{0} \in Y$, so that one obtains $X \backslash Y=F(n)$. It follows from (39) and (40) that one has a canonical isomorphism of abelian groups

$$
\mathcal{A}_{n}=H B(Y) \oplus H A(X / Y)=H B(Y) \times H A(X / Y)=H_{\phi}(X, Y)=H_{\phi}\left(\partial[n]^{*}\right) .
$$

Let $\theta \in \operatorname{Hom}_{\Delta}([m],[n])$, and $\mathcal{A}(\theta): \mathcal{A}_{n} \rightarrow \mathcal{A}_{m}$ as described in [25] §III.2, p. 160-161. We show that $\mathcal{A}(\theta)=H_{\phi} \circ \partial\left(\theta^{*}\right)$, where $\theta^{*}$ is defined in (45). By construction, $\mathcal{A}(\theta)$ is a morphism of abelian groups that is determined by its restriction to each copy $C_{j, \sigma}$ of $C_{j}$ indexed by a surjection $\sigma:[n] \rightarrow[j]$ as follows. One writes $\sigma \circ \theta$ uniquely as the composite $d \circ s$

$$
\sigma \circ \theta=d \circ s, \quad s \in \operatorname{Hom}_{\Delta}([m],[k]), d \in \operatorname{Hom}_{\Delta}([k],[j]),
$$

where $s$ is a surjection and $d$ an injection. Then, for $\alpha \in C_{j, \sigma}, \mathcal{A}(\theta)(\alpha)=\mathcal{A}(d)\left(\iota_{s, \sigma} \alpha\right)$, where $\iota_{s, \sigma}$ is the identity map of $C_{j}$ that identifies $C_{j, \sigma}$ with $C_{j, s}$. The map $\mathcal{A}(d)$ is the identity when $d=\operatorname{Id}$ (i.e. when $k=j$ ) and is 0 otherwise, unless $k=j-1$ and $d \in \operatorname{Hom}_{\Delta}([j-1],[j])$ is the injection which misses $j$. In the case of the complex $\mathfrak{C}$, the only indices are $j=0,1$. For $j=0$, there is only one element $\sigma:[n] \rightarrow[j], \sigma \circ \theta$ is surjective and is the only element of $\operatorname{Hom}_{\Delta}([m]$, [0]). This shows that the restriction of $\mathcal{A}(\theta)$ to $H B(Y)$ is the identity. For $j=1$, one has $\sigma \in F(n)$ i.e. $\sigma:[n] \rightarrow[1]$ is surjective. If the same result holds for $\sigma \circ \theta:[m] \rightarrow[1]$ then, by reasoning as above, one concludes that $\mathcal{A}(\theta)$ is the natural identification of $C_{1, \sigma}$ with $C_{1, \sigma \circ \theta}$. When $\sigma \circ \theta:[m] \rightarrow[1]$ fails to be surjective there are two cases to consider. One has either $\sigma \circ \theta=\xi_{0}$ or $\sigma \circ \theta=\xi_{m+1}$. The only case in which the obtained map $\mathcal{A}(\theta)$ is non-zero on $C_{1, \sigma}$ is when the morphism $d$ in the decomposition $\sigma \circ \theta=d \circ s$ of (48) is $\delta_{1} \in \operatorname{Hom}_{\Delta}([0],[1])$, with $\delta_{1}$ missing 1 . This happens 


\section{Alain Connes and Caterina Consani}

when $\sigma \circ \theta=\xi_{m+1}$ since it corresponds to a mono o epi decomposition of the form

$$
\sigma \circ \theta=\delta_{1} \circ \tau, \quad \delta_{1} \in \operatorname{Hom}_{\Delta}([0],[1]), \quad \tau \in \operatorname{Hom}_{\Delta}([m],[0] .
$$

In that case, $\mathcal{A}(\theta)$ restricted to $C_{1, \sigma}$ is the map to $C_{0}$ given by the boundary $\phi: C_{1} \rightarrow C_{0}$ of the complex $\mathfrak{C}$. By adding together these various contributions of $\mathcal{A}(\theta)$ one derives the following formula (using the identification (47) and the map $p: A \cup B \rightarrow B$ equal to $\phi$ on $A$ and the identity on $B$ )

$$
\mathcal{A}(\theta)(\psi)(y)= \begin{cases}\sum_{x \in X \mid x \circ \theta=y} \psi(x) & \text { if } y \notin Y \\ \sum_{x \in X \mid x \circ \theta=y} p(\psi(x)) & \text { if } y \in Y, y \neq *\end{cases}
$$

Since $\theta^{*}(x)=x \circ \theta$, one thus obtains $\mathcal{A}(\theta)=H_{\phi} \circ \partial\left(\theta^{*}\right)$ as required.

The covariant functor $H_{\phi}: \mathfrak{S e t s}_{2, *} \longrightarrow \mathfrak{A} \mathfrak{b}$, when composed with the Eilenberg-MacLane functor $H$ defines a functor $H H_{\phi}: \mathfrak{S e t s}_{2, *} \longrightarrow \mathbb{S}-\operatorname{Mod}$ whose explicit description is provided by the following

Lemma 4.6. The functor $H H_{\phi}: \mathfrak{S e t s}_{2, *} \longrightarrow \mathbb{S}-\operatorname{Mod}$ is naturally isomorphic to the functor $\left(\left(U \circ H_{\phi}\right) \times I d\right) \circ \gamma$, where $U: \mathfrak{A b} \longrightarrow \mathfrak{S e t s}_{*}$ is the forgetful functor and $\gamma: \mathfrak{S e t s}_{2, *} \longrightarrow \Gamma \mathfrak{S e t s}_{2, *}$ associates to an object $(X, Y)$ of $\mathfrak{S e t s}_{2, *}$ the covariant functor

$$
\gamma(X, Y): \Gamma^{\mathrm{op}} \longrightarrow \mathfrak{S e t s}_{2, *}, \quad k_{+} \mapsto\left(X \wedge k_{+}, Y \wedge k_{+}\right) .
$$

Proof. Both $H H_{\phi}=H \circ H_{\phi}$ and $\left(\left(U \circ H_{\phi}\right) \times \mathrm{Id}\right) \circ \gamma$ are functors from $\mathfrak{S e t s}_{2, *}$ to the category of $\mathbb{S}$ modules. More precisely, the functor $\left(\left(U \circ H_{\phi}\right) \times \mathrm{Id}\right) \circ \gamma$, obtained by applying $U \circ H_{\phi}$ "pointwise" takes values in the category $\Gamma \mathfrak{S e t s}_{*}$ of pointed $\Gamma$-sets that is the same as the category $\mathbb{S}-\operatorname{Mod}$. The functor $H_{\phi}$ fulfills, for any pointed map $\rho: k_{+} \rightarrow \ell_{+}$, the following equalities

$$
H_{\phi}\left(X \wedge k_{+}, Y \wedge k_{+}\right)=H_{\phi}(X, Y)^{k}, \quad H_{\phi}(\operatorname{Id} \wedge \rho)=H\left(H_{\phi}(X, Y), \rho\right) .
$$

Indeed, elements of $H_{\phi}\left(X \wedge k_{+}, Y \wedge k_{+}\right)$are by construction functions $\psi(x, j) \in A \cup B$, for $x \in X \backslash\{*\}$, $j \in\{1, \ldots, k\}$ with values in $A$ when $x \notin Y$, and in $B$ for $x \in Y$. Equivalently, these elements are described by $k$-tuples of functions $\psi_{j} \in H_{\phi}(X, Y)$, where $\psi_{j}(x):=\psi(x, j)$ for $x \in X \backslash\{*\}$ and $j \in\{1, \ldots, k\}$. The map $\tau=\operatorname{Id} \wedge \rho:\left(X \wedge k_{+}, Y \wedge k_{+}\right) \rightarrow\left(X \wedge \ell_{+}, Y \wedge \ell_{+}\right)$is a map of pairs that preserves the subset and also its complement. For such maps $\tau$ the formula (41) simplifies to

$$
H_{\phi}(\tau)=(H B(\tau), H A(\tau)) .
$$

This argument proves the second equality in (50). This equality gives the isomorphism of the S-modules $U \circ H_{\phi} \circ \gamma(X, Y) \simeq H H_{\phi}(X, Y)$ for a fixed $(X, Y)$. To complete the proof let us show that this isomorphism is a natural transformation of functors. Let $f:(X, Y) \rightarrow\left(X^{\prime}, Y^{\prime}\right)$ be a morphism in $\mathfrak{S e t s}_{2, *}$. One has $\gamma(f)=f \wedge$ Id as a morphism from $\left(X \wedge k_{+}, Y \wedge k_{+}\right)$to $\left(X^{\prime} \wedge k_{+}, Y^{\prime} \wedge k_{+}\right)$. Moreover

$$
H_{\phi}(f \wedge \mathrm{Id})=H_{\phi}(f)^{k}: H_{\phi}(X, Y)^{k} \rightarrow H_{\phi}\left(X^{\prime}, Y^{\prime}\right)^{k}
$$

follows directly from (44) using $(f \wedge \mathrm{Id})(x, j)=(f(x), j) \forall x \in X, j \in\{1, \ldots, k\}$. Finally, (51) shows that the identification (50) is a natural transformation of functors.

By combining Proposition 4.5 with Lemma 4.6 we finally obtain

Theorem 4.7. Let $\phi: A \rightarrow B$ be a morphism of abelian groups. The $\Gamma$-space associated by the Dold-Kan correspondence to the short complex $\mathfrak{C}$ in (36) is canonically isomorphic to the functor

$$
\left(\left(U \circ H_{\phi}\right) \times I d\right) \circ \gamma \circ \partial: \Delta^{\mathrm{op}} \longrightarrow \mathbb{S}-\operatorname{Mod} .
$$


Proof. By construction this $\Gamma$-space is obtained by applying the Eilenberg-MacLane functor $H$ to the simplicial abelian group associated by the Dold-Kan correspondence to the short complex $\mathfrak{C}$. Thus by Proposition 4.5 it is the functor $H H_{\phi} \circ \partial: \Delta^{\mathrm{op}} \longrightarrow \mathbb{S}-\operatorname{Mod}$. Lemma 4.6 then allows one to replace $H H_{\phi}$ by $\left(\left(U \circ H_{\phi}\right) \times \mathrm{Id}\right) \circ \gamma$ and this determines the required equality.

As indicated by Theorem 4.7, the construction of the $\Gamma$-space associated to the short complex $\mathfrak{C}$ in (36) by the Dold-Kan correspondence involves the morphism $\phi: A \rightarrow B$ of abelian groups only through the composite functor $U \circ H_{\phi}: \mathfrak{S e t s}_{2, *} \longrightarrow \mathfrak{S e t s}_{*}$. In the next section we show that the latter functor is still meaningful when $\phi: A \rightarrow B$ is replaced by the morphism (35) of $\mathbb{S}$-modules as in $\S 4.2$.

\subsection{The $\Gamma$-space $\mathbf{H}(D)$}

In this section we associate to an Arakelov divisor $D$ on $\overline{\operatorname{Spec} \mathbb{Z}}$ a $\Gamma$-space $\mathbf{H}(D)$ that embodies the Dold-Kan counterpart of the short complex (35) associated to the Riemann-Roch problem for the divisor. This construction is based on Theorem 4.7 and the following

Lemma 4.8. Let $L \subset \mathbb{R}$ be a discrete subgroup and $\lambda \in \mathbb{R}_{+}^{*}$. Let $\phi: \mathbb{R} \rightarrow \mathbb{R} / L$ be the quotient map. The following equality defines a subfunctor of $U \circ H_{\phi}: \mathfrak{S e t s}_{2, *} \longrightarrow \mathfrak{S e t s}_{*}$

$$
K_{L, \lambda}: \mathfrak{S e t s}_{2, *} \longrightarrow \mathfrak{S e t s}_{*}, \quad K_{L, \lambda}(X, Y):=\left\{\psi \in H_{\phi}(X, Y)\left|\sum_{x \in X \backslash Y}\right| \psi(x) \mid \leq \lambda\right\} .
$$

Proof. The setting (52) is well defined in view of (42), showing that $\psi(x) \in \mathbb{R}, \forall x \in X \backslash Y$. Let $f:(X, Y) \rightarrow\left(X^{\prime}, Y^{\prime}\right)$ be a morphism in $\mathfrak{S e t s}_{2, *}$, then by (44) one has

$$
H_{\phi}(f)(\psi)(y)=\sum_{x \in X, f(x)=y} \psi(x), \forall y \notin Y^{\prime} .
$$

The key fact here is that since $f:(X, Y) \rightarrow\left(X^{\prime}, Y^{\prime}\right)$ is a morphism of pairs, the following implication holds

$$
y \in X^{\prime} \backslash Y^{\prime} \& f(x)=y \Rightarrow x \in X \backslash Y,
$$

from which one derives the inequality

$$
\sum_{y \in X^{\prime} \backslash Y^{\prime}}\left|H_{\phi}(f)(\psi)(y)\right| \leq \sum_{x \in X \backslash Y}|\psi(x)| .
$$

This proves that $K_{L, \lambda}: \mathfrak{S e t s}_{2, *} \longrightarrow \mathfrak{S e t s}_{*}$ is a subfunctor of $U \circ H_{\phi}$.

With the notation of $\S 4.3$ and in particular as in Lemma 4.6, we have

Proposition 4.9. Let $D=\sum a_{j}\left\{p_{j}\right\}+a\{\infty\}$ be a divisor on $\overline{\operatorname{Spec} \mathbb{Z}}$ and let $\lambda=e^{a}$. Consider the abelian group

The functor

$$
L=H^{0}\left(\operatorname{Spec} \mathbb{Z}, \mathcal{O}\left(D_{\text {finite }}\right)\right) \subset \mathbb{Q} \subset \mathbb{R}
$$

$$
\mathbf{H}(D):=\left(K_{L, \lambda} \times I d\right) \circ \gamma \circ \partial: \Delta^{\mathrm{op}} \longrightarrow \mathbb{S}-\operatorname{Mod}
$$

defines a $\Gamma$-space that depends only on the linear equivalence class of $D$.

Proof. The sheaf $\mathcal{O}\left(D_{\text {finite }}\right)$ on Spec $\mathbb{Z}$ is viewed as a subsheaf of the constant sheaf $\mathbb{Q}$ : this gives meaning to the inclusion $L=H^{0}\left(\operatorname{Spec} \mathbb{Z}, \mathcal{O}\left(D_{\text {finite }}\right)\right) \subset \mathbb{Q}$. Let $q \in \mathbb{Q}_{+}^{*}$ and $D^{\prime}=D+(q)$, then $L^{\prime}=q^{-1} L$ and $\lambda^{\prime}=q^{-1} \lambda$. The multiplication by $q^{-1}$ induces an isomorphism of functors $K_{L, \lambda} \rightarrow K_{L^{\prime}, \lambda^{\prime}}$. This shows that $\mathbf{H}(D)$ only depends on the equivalence class of $D$ modulo principal divisors. 


\section{Alain Connes and Caterina Consani}

\section{5 $\mathbf{H}(D)$ at level 1}

When evaluated on the object $1_{+}$of $\Gamma^{\mathrm{op}}$, the $\Gamma$-space $\mathbf{H}(D)$ defines a simplicial set $\mathbf{H}(D)\left(1_{+}\right)$. By construction, this is a sub-simplicial set of the Kan simplicial set associated to the morphism of abelian groups (viewed as a short complex) $\phi: \mathbb{R} \rightarrow \mathbb{R} / L$, however 'per-se' it is not a Kan simplicial set. Thus one needs to exert care when considering the homotopy of $\mathbf{H}(D)\left(1_{+}\right)$: we refer to [20] §2.1, for the general notions and notation used here as follows, when referring to the homotopies. We simply recall that the new homotopy theory $\pi_{n}^{\text {new }}$ introduced in op.cit. is reduced to $\pi_{0}^{\text {new }}$ by implementing the endofunctor $\Omega$ of "decalage" in the category $\mathcal{S}_{\star}$ of pointed simplicial sets, as follows

$$
\pi_{n}^{\text {new }}(X, \star):=\pi_{0}^{\text {new }}\left(\Omega^{n}(X)\right) .
$$

One obtains, in this way, a concrete description of the elements of $\pi_{n}^{\text {new }}(X, \star)$ and of the following homotopy relation:

(i) The 0 -skeleton $\left(\Omega^{n}(X)\right)_{0}$ is the set of simplices $x \in X_{n}$ such that $\partial_{j}(x)=* \forall j$.

(ii) $\left(\Omega^{n}(X)\right)_{0}$ coincides with $\operatorname{Hom}_{\mathcal{S}_{*}}\left(S^{n}, X\right) \subset X_{n}$, where $S^{n}$ is obtained by collapsing the boundary $\partial \Delta[n]$ of the standard simplex to a single base point.

(iii) The relation $\mathcal{R}$ on $\left(\Omega^{n}(X)\right)_{0}=\operatorname{Hom}_{\mathcal{S}_{*}}\left(S^{n}, X\right) \subset X_{n}$ given by

$$
x \mathcal{R} y \Longleftrightarrow \exists z \in\left(\Omega^{n}(X)\right)_{1} \text { s.t. } \partial_{0} z=x \text { and } \partial_{1} z=y
$$

coincides with the relation of homotopy between $n$-simplices as in the following formula

$$
\mathcal{R}=\left\{(x, y) \in X_{n} \times X_{n}\left|\partial_{j} x=\partial_{j} y \forall j \& \exists z\right| \partial_{j} z=s_{n-1} \partial_{j} x \forall j<n, \partial_{n} z=x, \partial_{n+1} z=y\right\} .
$$

When working with this (new) homotopies $\pi_{n}$ usually it happens that the relation $\mathcal{R}$ as in (56) fails to be an equivalence relation, since the pointed simplicial sets fail in general to be fibrant. Even though (the new) $\pi_{0}$ cannot be directly described as a quotient, we can still specify its cardinality, using the following general definition

Definition 4.10. Let $\mathcal{R}$ be a reflexive relation on a set $E$. Define $\#(E / \mathcal{R})$ to be the maximal cardinality of subsets $F \subset E$ such that $x, y \in F \& x \neq y \Rightarrow(x, y) \notin \mathcal{R}$.

Obviously, this definition determines the cardinality of the quotient $E / \mathcal{R}$ when $\mathcal{R}$ is an equivalence relation. As an example, consider the relation $d(x, y) \leq \epsilon$ in a metric space $(E, d)$. Then the number $\#(E / \mathcal{R})$ is called the packing number and is denoted by $\mathcal{M}(\epsilon)$. It plays an important role in the notion of $\epsilon$-capacity [30].

The following proposition supplies in particular a first approximation on the definition and the cardinality of $H^{1}(\overline{\operatorname{Spec} \mathbb{Z}}, \mathcal{O}(D))$.

Proposition 4.11. The simplicial set $\mathbf{H}(D)\left(1_{+}\right)$fulfills the following properties:

(i) $\pi_{0}\left(\mathbf{H}(D)\left(1_{+}\right)\right)=*$ for $\operatorname{deg} D \geq-\log 2$.

(ii) For $\operatorname{deg} D<0$, \# $\left(\pi_{0}\left(\mathbf{H}(D)\left(1_{+}\right)\right)\right)$is the largest integer $n<e^{-\operatorname{deg} D}$.

(iii) $\pi_{1}\left(\mathbf{H}(D)\left(1_{+}\right)\right)$is in canonical bijection with the global sections $H^{0}(\overline{\operatorname{Spec} \mathbb{Z}}, \mathcal{O}(D))$.

(iv) $\pi_{n}\left(\mathbf{H}(D)\left(1_{+}\right)\right)$is trivial for $n>1$.

Proof. (i) We can assume, by working up-to linear equivalence, that $D_{\text {finite }}=0$ so that $D=$ $a\{\infty\}$ with $a=\operatorname{deg} D$. Then, $L=H^{0}\left(\operatorname{Spec} \mathbb{Z}, \mathcal{O}\left(D_{\text {finite }}\right)\right)=\mathbb{Z} \subset \mathbb{R}$. By construction, the pointed simplicial set $\mathbf{H}(D)\left(1_{+}\right)$is obtained by composing the functor $K_{L, \lambda}: \mathfrak{S e t s}_{2, *} \longrightarrow \mathfrak{S e t s}_{*}\left(\lambda=e^{a}\right)$ 
with $\partial: \Delta^{\mathrm{op}} \longrightarrow \mathfrak{S e t s}_{2, *}$. In degree 0 and with the notation of (38), it is described for the $\xi_{j} \in \operatorname{Hom}_{\Delta}([n],[1]), 0 \leq j \leq n+1$, by

$$
K_{L, \lambda}\left(\partial[0]^{*}\right)=K_{L, \lambda}\left(\left(*, \xi_{1}\right),\left(*, \xi_{1}\right)\right)=\mathbb{R} / L .
$$

In degree 1 it is given by

$$
K_{L, \lambda}\left(\partial[1]^{*}\right)=K_{L, \lambda}\left(\left(*, \xi_{1}, \xi_{2}\right),\left(*, \xi_{2}\right)\right)=\left\{\left(\psi_{1}, \psi_{2}\right) \in \mathbb{R} \times \mathbb{R} / L|| \psi_{1} \mid \leq \lambda\right\} .
$$

One has $\xi_{0} \circ \delta_{j}=\xi_{0}$ for all $j=0,1$ and

$$
\xi_{1} \circ \delta_{0}=\xi_{0}, \quad \xi_{2} \circ \delta_{0}=\xi_{1}, \quad \xi_{1} \circ \delta_{1}=\xi_{1}, \quad \xi_{2} \circ \delta_{1}=\xi_{1} .
$$

Thus, with $\partial_{j}: K_{L, \lambda}\left(\partial[1]^{*}\right) \rightarrow K_{L, \lambda}\left(\partial[0]^{*}\right)$ the corresponding boundaries, one gets

$$
\partial_{0}(\psi)=\psi_{2}, \quad \partial_{1}(\psi)=\psi_{1}+\psi_{2}, \quad \forall \psi=\left(\psi_{1}, \psi_{2}\right) \in K_{L, \lambda}\left(\partial[1]^{*}\right) .
$$

This argument shows that the relation defined on elements of $K_{L, \lambda}\left(\partial[0]^{*}\right)$ by

$$
\mathcal{R}(\alpha, \beta) \Longleftrightarrow \exists \psi \in K_{L, \lambda}\left(\partial[1]^{*}\right) \mid \partial_{0}(\psi)=\alpha \& \partial_{1}(\psi)=\beta
$$

is, in general, not an equivalence relation. The relation $\mathcal{R}$ of (59) is described, using (58) as follows:

$$
\left(\psi, \psi^{\prime}\right) \in \mathcal{R} \subset \mathbb{R} / L \times \mathbb{R} / L \Longleftrightarrow \exists \alpha \in \mathbb{R},|\alpha| \leq \lambda, \psi^{\prime}=\psi+\alpha .
$$

When $\lambda \geq 1 / 2$, i.e. $\operatorname{deg} D \geq-\log 2$ this relation becomes the equivalence relation with quotient a single element.

(ii) With the notations of (i), \#( $\left.\pi_{0}\left(\mathbf{H}(D)\left(1_{+}\right)\right)\right)$is the packing number of the metric space $\mathbb{R} / \mathbb{Z}$ (endowed with the quotient metric of the usual metric on $\mathbb{R}$ ) for the constant $\lambda$. For any subset fulfilling the separation condition of Definition 4.10, the balls of radius $\lambda / 2$ are pairwise disjoint and hence $n \lambda<1$. Conversely, if $n \lambda<1$ the $n$ points $x_{j}=\frac{j}{n}, 0 \leq j<n$ are such that, in the metric space $\mathbb{R} / \mathbb{Z}, d\left(x_{i}, x_{j}\right)>\lambda$ for $i \neq j$. This shows (ii).

(iii) With the above notations, $\mathbf{H}(D)\left(1_{+}\right)$is described in degree 1 by (57) and by (58) one has: $\partial_{0}(\psi)=\psi_{2}, \quad \partial_{1}(\psi)=\psi_{1}+\psi_{2}$. Thus the spherical conditions $\partial_{j}(\psi)=*$ mean that $\psi_{2}=0$ and that the class of $\psi_{1}$ in $\mathbb{R} / \mathbb{Z}$ is zero i.e. $\psi_{1} \in L=\mathbb{Z}$. Hence the spherical elements are in canonical bijection with the global sections $H^{0}(\overline{\operatorname{Spec} \mathbb{Z}}, \mathcal{O}(D))$. It remains to describe the relation $(56)$ between the spherical elements. With $X_{n}=K_{L, \lambda}\left(\partial[n]^{*}\right)$, this is

$$
\mathcal{R}=\left\{\left(\phi, \phi^{\prime}\right) \in X_{1} \times X_{1}\left|\partial_{j} \phi=\partial_{j} \phi^{\prime}=* \forall j \& \exists \psi \in X_{2}\right| \partial_{0} \psi=*, \partial_{1} \psi=\phi, \partial_{2} \psi=\phi^{\prime}\right\} .
$$

$K_{L, \lambda}$ is described in degree 2 by

$$
K_{L, \lambda}\left(\partial[2]^{*}\right)=K_{L, \lambda}\left(\left(*, \xi_{1}, \xi_{2}, \xi_{3}\right),\left(*, \xi_{3}\right)\right)=\left\{\left(\psi_{1}, \psi_{2}, \psi_{3}\right) \in \mathbb{R} \times \mathbb{R} \times \mathbb{R} / L|| \psi_{1}|+| \psi_{2} \mid \leq \lambda\right\} .
$$

The three boundary maps take values in $\mathbb{R} \times \mathbb{R} / L$ and are given by

$$
\partial_{0}(\psi)=\left(\psi_{2}, \psi_{3}\right), \partial_{1}(\psi)=\left(\psi_{1}+\psi_{2}, \psi_{3}\right), \partial_{2}(\psi)=\left(\psi_{1}, \psi_{2}+\psi_{3}\right) .
$$

The condition $\partial_{0}(\psi)=*$ of $(60)$ implies that $\psi_{2}=0$. Hence the relation coming from the pairs $\left(\partial_{1}(\psi), \partial_{2}(\psi)\right)$ is the identity. This proves (iii).

(iv) For $n>1$ one has

$$
K_{L, \lambda}\left(\partial[n]^{*}\right)=\left\{\left(\psi_{j}\right)_{j \in\{1, \ldots, n\}} \in \mathbb{R}^{n-1} \times \mathbb{R} / L\left|\sum_{j \in\{1, \ldots, n-1\}}\right| \psi_{j} \mid \leq \lambda\right\} .
$$




\section{Alain Connes and Caterina Consani}

The boundaries $\partial_{j}: K_{L, \lambda}\left(\partial[n]^{*}\right) \rightarrow K_{L, \lambda}\left(\partial[n-1]^{*}\right)$ are given, for $j \in\{0, \ldots, n\}$, as follows

$$
\left(\partial_{j} \psi\right)_{i}= \begin{cases}\psi_{i} & \text { if } i<j \\ \psi_{j}+\psi_{j+1} & \text { if } i=j \\ \psi_{j+1} & \text { if } i>j\end{cases}
$$

Thus, for instance for $n=3$ one obtains

$$
\left(\begin{array}{c}
\partial_{0} \psi \\
\partial_{1} \psi \\
\partial_{2} \psi \\
\partial_{3} \psi
\end{array}\right)=\left(\begin{array}{ccc}
\psi_{2} & \psi_{3} & \psi_{4} \\
\psi_{1}+\psi_{2} & \psi_{3} & \psi_{4} \\
\psi_{1} & \psi_{2}+\psi_{3} & \psi_{4} \\
\psi_{1} & \psi_{2} & \psi_{3}+\psi_{4}
\end{array}\right)
$$

Hence, for $n>1$ the only solution of the spherical condition $\partial_{j} \psi=0 \forall j$ is $\psi=0$. Indeed, the condition $\partial_{0} \psi=0$ implies that $\psi_{i}=0$ for all $i>1$ and the condition $\partial_{2} \psi=0$ shows that $\psi_{1}=0$.

\subsection{The higher levels of $\mathbf{H}(D)$}

One can compute the precise number of elements of $\|H \mathbb{Z}\|_{\lambda}\left(k_{+}\right)$. We set

$$
\gamma(n, k):=\#\left(\|H \mathbb{Z}\|_{n}\left(k_{+}\right)\right) .
$$

Any element $\phi$, with $\sum|\phi(x)| \leq n$, has a support $s(\phi) \subseteq\{1, \ldots, k\}$. With the exception of the single element 0 , we let $m>0$ be the size of the support $s(\phi)=\left\{x_{1}, x_{2}, \ldots, x_{m}\right\}$, where the elements are listed in increasing order. This way, one obtains a list of numbers all $\leq n$ that is equivalently described by the subset (with $\psi=|\phi|$ )

$$
\left\{\psi\left(x_{1}\right), \psi\left(x_{1}\right)+\psi\left(x_{2}\right), \ldots, \psi\left(x_{1}\right)+\psi\left(x_{1}\right)+\ldots+\psi\left(x_{m}\right)\right\} \subset\{1, \ldots, n\} .
$$

This process gives, for each of the $\left(\begin{array}{c}k \\ m\end{array}\right)$ subsets with $m$ elements, the number $2^{m}\left(\begin{array}{c}n \\ m\end{array}\right)$ of possible choices, where the factor $2^{m}$ takes into account the signs of the $\phi(x)$. Thus one obtains the hypergeometric function

$$
\gamma(n, k)=1+\sum_{m=1}^{k} 2^{m}\left(\begin{array}{c}
k \\
m
\end{array}\right)\left(\begin{array}{l}
n \\
m
\end{array}\right)={ }_{2} F_{1}(-k,-n ; 1 ; 2) .
$$

Using the vanishing of binomial coefficients, one can replace the index $k$ in the above sum by the $\inf (k, n)$ showing the symmetry

$$
\gamma(n, k)=\gamma(k, n)
$$

Next proposition displays the properties of the higher levels $\pi_{n}\left(\mathbf{H}(D)\left(k_{+}\right)\right)$

Proposition 4.12. Let $D=\sum a_{j}\left\{p_{j}\right\}+a\{\infty\}$ be a divisor on $\overline{\text { Spec } \mathbb{Z}}$. The following facts hold

(i) $\pi_{1}(\mathbf{H}(D))=\|H L\|_{e^{a}}$, as $\mathbb{S}$-modules, with $L=H^{0}\left(\operatorname{Spec} \mathbb{Z}, \mathcal{O}\left(D_{\text {finite }}\right)\right)$.

(ii) $\# \pi_{1}\left(\mathbf{H}(D)\left(k_{+}\right)\right)=\gamma(n, k)$ for $n=\operatorname{IntegerPart}\left(e^{\operatorname{deg}(D)}\right)$.

(iii) $\pi_{0}\left(\mathbf{H}(D)\left(k_{+}\right)\right)=* \Longleftrightarrow k \leq 2 e^{\operatorname{deg}(D)}$.

(iv) $\pi_{n}\left(\mathbf{H}(D)\left(k_{+}\right)\right)$is trivial for $n>1$.

Proof. ( $i$ ) The simplicial set $\mathbf{H}(D)\left(k_{+}\right)$is described in degree 0, with the notation (38) for the $\xi_{j} \in \operatorname{Hom}_{\Delta}([n],[1]), 0 \leq j \leq n+1$, and with $\lambda=e^{a}$, by

$$
K_{L, \lambda}\left(\partial[0]^{*} \wedge k_{+}\right)=K_{L, \lambda}\left(\left(*, \xi_{1}\right) \wedge k_{+},\left(*, \xi_{1}\right) \wedge k_{+}\right)=(\mathbb{R} / L)^{k} .
$$

In degree 1 it is described by

$$
K_{L, \lambda}\left(\partial[1]^{*} \wedge k_{+}\right)=K_{L, \lambda}\left(\left(*, \xi_{1}, \xi_{2}\right) \wedge k_{+},\left(*, \xi_{2}\right) \wedge k_{+}\right)=\|H \mathbb{R}\|_{\lambda}\left(k_{+}\right) \times(\mathbb{R} / L)^{k} .
$$


The corresponding boundaries $\partial_{j}: K_{L, \lambda}\left(\partial[1]^{*} \wedge k_{+}\right) \rightarrow K_{L, \lambda}\left(\partial[0]^{*} \wedge k_{+}\right)$are

$$
\partial_{0}(\psi)=\psi_{2}, \quad \partial_{1}(\psi)=\psi_{1}+\psi_{2}, \quad \forall \psi=\left(\psi_{1}, \psi_{2}\right) \in K_{L, \lambda}\left(\partial[1]^{*} \wedge k_{+}\right) .
$$

The spherical conditions $\partial_{j}(\psi)=0$ mean that $\psi_{2}=0 \in(\mathbb{R} / L)^{k}$ and that the image of $\psi_{1} \epsilon$ $\|H \mathbb{R}\|_{\lambda}\left(k_{+}\right)$is zero in $(\mathbb{R} / L)^{k}$. Thus $\psi_{1} \in\|H L\|_{\lambda}\left(k_{+}\right)$. Moreover the relation (56) between the spherical elements is trivial for the same reason as stated in the proof of Proposition 4.11 (iii). (ii) follows from $(i)$.

(iii) The relation $\mathcal{R}$ of $(56)$ on $K_{L, \lambda}\left(\partial[0]^{*} \wedge k_{+}\right)=(\mathbb{R} / L)^{k}$ is given by

$$
\left(\phi, \phi^{\prime}\right) \in \mathcal{R} \Longleftrightarrow \exists \psi \in\|H \mathbb{R}\|_{\lambda}\left(k_{+}\right) \text {s. t. } \phi^{\prime}=\phi+\psi \text {. }
$$

Let $d$ be the metric on $\mathbb{R} / L$ which is induced by the usual distance on $\mathbb{R}$. Then

$$
\left(\phi, \phi^{\prime}\right) \in \mathcal{R} \Longleftrightarrow \sum d\left(\phi_{j}, \phi_{j}^{\prime}\right) \leq \lambda .
$$

Thus the relation $\mathcal{R}$ is equal to the square of $(\mathbb{R} / L)^{k}$, i.e. all elements are equivalent if and only if the sum of the diameters of the metric spaces $\mathbb{R} / L$ is less than $\lambda$, i.e. $k / 2 \leq e^{\operatorname{deg}(D)}$ (one can assume that $D=a\{\infty\}$ so that $L=\mathbb{Z}$ and $\left.\lambda=e^{\operatorname{deg}(D)}\right)$.

(iv) For $n>1$ one has

$$
K_{L, \lambda}\left(\partial[n]^{*} \wedge k_{+}\right)=\left\{\left(\psi_{j}\right)_{j \in\{1, \ldots, n\}} \in\left(\mathbb{R}^{k}\right)^{n-1} \times(\mathbb{R} / L)^{k} \mid \sum_{j \in\{1, \ldots, n-1\}}\left\|\psi_{j}\right\| \leq \lambda\right\},
$$

where the norm of vectors in $\mathbb{R}^{k}$ is the $\ell^{1}$-norm. The boundaries $\partial_{j}: K_{L, \lambda}\left(\partial[n]^{*} \wedge k_{+}\right) \rightarrow$ $K_{L, \lambda}\left(\partial[n-1]^{*} \wedge k_{+}\right)$are given for $j \in\{0, \ldots, n\}$ by

$$
\left(\partial_{j} \psi\right)_{i}= \begin{cases}\psi_{i} & \text { if } i<j \\ \psi_{j}+\psi_{j+1} & \text { if } i=j \\ \psi_{j+1} & \text { if } i>j\end{cases}
$$

Thus the same argument of the proof of Proposition 4.11 shows that, for $n>1$ there is no non-zero solution of the spherical condition.

Proposition $4.12(i)$ shows that the $\mathbb{S}$-module $\pi_{1}(\mathbf{H}(D))$ qualifies as the module $H^{0}(\overline{\operatorname{Spec} \mathbb{Z}}, \mathcal{O}(D))$ of global sections of $\mathcal{O}(D)$. Before identifying $H^{1}(\overline{\operatorname{Spec} \mathbb{Z}}, \mathcal{O}(D))$ with $\pi_{0}(\mathbf{H}(D))$ one needs to take care of the relation $\mathcal{R}$ defining the homotopy among elements of $\pi_{0}$, since $\mathcal{R}$ is not in general an equivalence relation. To handle this difficulty one can implement the topos $\mathfrak{S e t s}^{(2)}$ introduced in [20] (and known as the topos of reflexive (directed) graphs) in order to keep the full information supplied by the relation.

\section{REFERENCES}

1 G. Almkvist, Endomorphisms of finitely generated projective modules over a commutative ring, Arkiv för Matematik Volume 11, Numbers 1-2 (1973), 263-301.

2 G. Almkvist, The Grothendieck ring of the category of endomorphisms, J. of Algebra 28,(1974), 375388.

3 M. F. Atiyah, D.O Tall, Group representations, $\lambda$-rings and the $J$-homomorphism. Topology 81969 253-297.

4 Borger, James (2009), $\Lambda$-rings and the field with one element, arXiv:0906.3146

5 J. B. Bost Theta invariants of euclidean lattices and infinite-dimensional hermitian vector bundles over arithmetic curves 


\section{Alain Connes and Caterina Consani}

6 A. Connes, Cohomologie cyclique et foncteurs Ext ${ }^{n}$. C. R. Acad. Sci. Paris Sér. I Math. 296 (1983), no. $23,953-958$.

7 A. Connes, C. Consani, Schemes over $\mathbb{F}_{1}$ and zeta functions, Compositio Mathematica 146 (6), (2010) $1383-1415$.

8 A. Connes, C. Consani On the notion of geometry over $\mathbb{F}_{1}$, (English summary) J. Algebraic Geom. 20 (2011), no. 3, 525-557.

9 A. Connes, C. Consani, Cyclic homology, Serre's local factors and the $\lambda$-operations, J. K-Theory 14 (2014), no. 1, 1-45

10 A. Connes, C. Consani, On the arithmetic of the BC-system. J. Noncommut. Geom. 8 (2014), no. 3, 873-945.

11 A. Connes, C. Consani, Projective geometry in characteristic one and the epicyclic category, Nagoya Mathematical Journal 217 (2015), 95-132.

12 A. Connes, C. Consani, The cyclic and epicyclic sites. Rend. Semin. Mat. Univ. Padova 134 (2015), $197-237$.

13 A. Connes, C. Consani, The Arithmetic Site, Comptes Rendus Mathématiques Ser. I 352 (2014), 971-975.

14 A. Connes, C. Consani, Geometry of the Arithmetic Site. Adv. Math. 291 (2016), 274-329.

15 A. Connes, C. Consani, The Scaling Site, C. R. Math. Acad. Sci. Paris 354 (2016), no. 1, 1-6.

16 A. Connes, C. Consani, Geometry of the Scaling Site, Selecta Math. (N.S.) 23 (2017), no. 3, 1803-1850.

17 A. Connes, C. Consani, Homological Algebra in Characteristic One, Higher Structures Journal 3 (2019), no. 1, 155-247.

18 A. Connes, C. Consani, The Riemann-Roch strategy, Complex lift of the Scaling Site, to appear on "Advances in Noncommutative Geometry, On the Occasion of Alain Connes' 70th Birthday", Springer (2020).

19 A. Connes, C. Consani, Absolute algebra and Segal's Gamma sets, J. Number Theory 162 (2016), $518-551$.

20 A. Connes, C. Consani, $\overline{\mathrm{Spec} \mathbb{Z}}$ and the Gromov norm, Theory and Applications of Categories, 35, No. 6, 2020, pp. 155178.

21 A. Connes, C. Consani, On Absolute Algebraic Geometry, the affine case, Preprint (2019). Available at https://arxiv.org/abs/1909.09796

22 A. Deitmar, Schemes over F1, in Number Fields and Function Fields, Two parallel worlds. Progr. in Math, vol. 239, (2005).

23 Dundas, Bjorn Ian; Goodwillie, Thomas G.; McCarthy, Randy The local structure of algebraic Ktheory. Algebra and Applications, 18. Springer-Verlag London, Ltd., London, 2013.

24 N. Durov, New approach to Arakelov geometry, arXiv:0704.2030

25 Goerss, Paul G.; Jardine, John F. Simplicial homotopy theory. Progress in Mathematics, 174. Birkhauser Verlag, Basel, 1999.

26 T. Goodwillie, A Topologist's View of Cyclic Homology. Math. Forschungsinst. Oberwolfach Tagungsber., 26/1987, Zyklische Kohomologie und ihre Anwendungen, 14-6/20-6, 1987.

27 M. Kapranov and A. Smirnov, Cohomology determinants and reciprocity laws Prepublication.

28 K. Kato Toric Singularities American Journal of Mathematics, Vol. 116, No. 5 (Oct., 1994), 1073-1099.

29 N. Kurokawa, H. Ochiai, A. Wakayama, Absolute Derivations and Zeta Functions Documenta Math. Kazuya Kato's Fiftieth Birthday (2003) 565-584.

30 Kolmogorov, A. N.; Tihomirov, V. M. $\epsilon$-entropy and $\epsilon$-capacity of sets in functional space. Amer. Math. Soc. Transl. (2) 171961 277-364.

31 J.L. Loday, Cyclic homology. Grundlehren der Mathematischen Wissenschaften, 301. Springer-Verlag, Berlin, 1998. 
32 Y. I. Manin, Lectures on zeta functions and motives (according to Deninger and Kurokawa) Columbia University Number-Theory Seminar (1992), Astérisque No. 228 (1995), 4, 121-163.

33 Mathai, D. Quillen Superconnections, Thom classes, and equivariant differential forms. Topology 25 (1986), 1, 85-110.

34 C. Soulé, Les variétés sur le corps à un élément. Mosc. Math. J. 4 (2004), no. 1, 217-244.

35 J. Tits, Sur les analogues algébriques des groupes semi-simples complexes. Colloque d'algèbre supérieure, Bruxelles 19-22 décembre 1956, Centre Belge de Recherches Mathématiques Établissements Ceuterick, Louvain; Librairie Gauthier-Villars, Paris (1957), 261-289.

36 B. Töen, M. Vaquié Au dessous de $\operatorname{Spec}(\mathbb{Z})$. J. K-Theory 3 (2009), no. 3, 437-500.

37 G. van der Geer, R. Schoff Effectivity of Arakelov Divisors and the Theta Divisor of a Number Field, Selecta Math. (N.S.) 6 (2000), no. 4, 377-398.

38 C. Wilkerson, Lambda-rings, binomial domains, and vector bundles over $C P^{\infty}$. Comm. Algebra 10 (1982), no. 3, 311-328.

Alain Connes alain@connes.org

College de France, I.H.E.S. and Ohio State University

Caterina Consani kc@math.jhu.edu

Department of Mathematics, The Johns Hopkins University

Baltimore, MD 21218 USA 